Review Article

\title{
Holographic Multiquarks in the Quark-Gluon Plasma: A Review
}

\author{
Piyabut Burikham ${ }^{1,2}$ and Ekapong Hirunsirisawat ${ }^{1}$ \\ ${ }^{1}$ Theoretical High-Energy Physics and Cosmology Group, Department of Physics, Faculty of Science, \\ Chulalongkorn University, Bangkok 10330, Thailand \\ ${ }^{2}$ Thailand Center of Excellence in Physics, CHE, Ministry of Education, Bangkok 10400, Thailand \\ Correspondence should be addressed to Piyabut Burikham, piyabut@gmail.com
}

Received 1 July 2011; Accepted 23 September 2011

Academic Editor: Ian Jack

Copyright (C) 2011 P. Burikham and E. Hirunsirisawat. This is an open access article distributed under the Creative Commons Attribution License, which permits unrestricted use, distribution, and reproduction in any medium, provided the original work is properly cited.

\begin{abstract}
We review the holographic multiquark states in the deconfined quark-gluon plasma. Nuclear matter can become deconfined by extremely high temperature and/or density. In the deconfined nuclear medium, bound states with colour degrees of freedom are allowed to exist. Using holographic approach, the binding energy and the screening length of the multiquarks can be calculated. Using the deconfined Sakai-Sugimoto model, the phase diagram of the multiquark phase, the vacuum phase, and the chiral-symmetric quark-gluon plasma can be obtained. Then we review the magnetic properties of the multiquarks and their phase diagrams. The multiquark phase is compared with the pure pion gradient, the magnetized vacuum, and the chiral-symmetric quark-gluon plasma phases. For moderate temperature and sufficiently large density at a fixed magnetic field, the mixed phase of multiquark and pion gradient is the most energetically preferred phase.
\end{abstract}

\section{Introduction}

At low energy, only hadrons can be observed. Due to the large coupling of the strong interaction on large distance scale, the genuine constituents of the nuclear matter are confined within the baryons and mesons. They can be explored only with a high energy probe, for example, in the deep inelastic scattering (DIS) experiments. When the energy scale involved is sufficiently large, roughly few hundred MeVs, the interaction among quarks and gluons become perturbatively weak, the phenomenon known as the asymptotic freedom. The quarks and gluons subsequently become "deconfined" from the confinement of the strong interaction.

For effectively free quarks and gluons, perturbative treatment of the Quantum Chromodynamics (QCD) has been proven very successful in making verifiable quantitative and reliable predictions. The QCD background calculations of the scattering of quarks 
and gluons at the Tevatron give accurate and vital results, which are crucial in providing the benchmark for the search of New Physics beyond the Standard Model. Nevertheless, unexpected ridge-like signals related to the strong interaction are already observed from the collision of proton and proton at the Large Hadron Collider (LHC) [1]. Further investigations are required in order to determine whether this ridge structure could be explained by the perturbative QCD or if it is nonperturbative in nature.

A general picture of the deconfinement process of the quarks and gluons within hadrons is currently incomplete at the most. Naively, from argument of the RGE (Renormalization Group Equation) running of the beta function, effectively free quarks and gluons are expected to appear at high energies and/or temperatures. Transition from nonperturbative phase of nuclear matter to the perturbative regime, where the perturbative QCD is reliable, is explored most successfully in the lattice approach. Lattice studies of the QCD predicts the deconfinement temperature around $175 \mathrm{MeV}$ [2]. Nuclear matter at such temperature would undergo a phase transition into a deconfined phase called the quarkgluon plasma (QGP). Most bound states of light quarks would melt down at this temperature leaving free quarks and gluons in the plasma. Remarkably, the mesonic states of heavy quarks (e.g., charmonium) in the nuclear matter at such high temperature tend to persist melting at least until $1.5 T_{c}$ [3-5] due to the remaining screened Coulomb-type binding potential between quark and antiquark. Multiquark states such as baryons can also exist in the QGP up to certain temperatures provided that the baryonic charge density is sufficiently large.

In the confined phase, only colour singlet states can exist as free particle due to the confinement. Above the deconfinement, quarks and gluons with colour charges can propagate with more freedom in the plasma. It is therefore possible that the coloured multiquark states such as diquarks could also exist in the deconfined nuclear medium. Similar to the mesonic states of the heavy quarks, these multiquarks could persist melting up to relatively high temperature above the deconfinement. We can expect the multiquarks to be abundant in the nuclear matter when the density is large up to temperature well above the deconfinement temperature. Consequently, it is interesting to investigate the physical properties of the multiquarks as well as their thermodynamical phase diagram in details. Unfortunately, perturbative QCD based on quarks and gluons is not reliable during the deconfinement phase transition. Lattice QCD is applicable only when the baryon density is small.

An alternative approach to study the strongly coupled gauge theory is the holographic model based on the AdS/CFT correspondence [6, 7]. A string theory in the curved background generated by D-branes source is conjectured to be dual to the gauge theory on the branes. The duality suggests a correspondence between the strongly coupled gauge theory on the branes and the weakly coupled string theory in the bulk. Extension of the duality to the finite temperature gauge theory can be done by adding a black hole horizon to the near-horizon limit of the background spacetime [8]. Baryons and multiquarks can be holographically constructed using the baryon vertex and strings [9-12].

In this paper, we will review the physics of the holographic multiquarks in the quark-gluon plasma using mainly the Sakai-Sugimoto (SS) model [13, 14]. The SS model and the holographic setup of the multiquarks is discussed in Section 2. Section 3 describes the thermodynamical properties and the phase diagram of the multiquark nuclear phase. Magnetic properties of the multiquark phase and the corresponding phase diagram are discussed in Sections 4 and 5, respectively. Section 6 concludes our paper. To present the main results of this paper, a summary table of the deconfined nuclear phase in the SS model is given in Table 1. 
Table 1: Summary table of the phases in the deconfined Sakai-Sugimoto model, $B$ represents the external magnetic field.

\begin{tabular}{|c|c|c|c|c|}
\hline$B=0$ & vacuum & \multicolumn{2}{|c|}{ Multiquark (MQ) } & $x$ S-QGP \\
\hline \multirow[t]{2}{*}{$\begin{array}{l}\text { Region in parameter } \\
\text { space }\end{array}$} & $d=0$ (i.e., $\left.\mu<\mu_{\text {source }}\right)$ & \multicolumn{2}{|c|}{$d>0$ (i.e., $\mu \geq \mu_{\text {source }}$ ) } & $d>0$ \\
\hline & & \multicolumn{2}{|c|}{$0 \leq n_{s} \lesssim 0.3$} & \\
\hline Preferred at & low $\mu$, low $T$ & \multicolumn{2}{|c|}{ High $\mu$, low $T$} & high $T$ \\
\hline $\begin{array}{l}\text { Important } \\
\text { properties }\end{array}$ & & \multicolumn{2}{|c|}{ Mixing of different $n_{s}$-multiquarks } & \\
\hline$B \neq 0$ & magnetized vacuum & $\nabla \varphi$ & $\mathrm{MQ}-\nabla \varphi$ & $x^{S-Q G P}$ \\
\hline \multirow{5}{*}{$\begin{array}{l}\text { Region in } \\
\text { parameter space }\end{array}$} & $\mu_{\text {source }}=0$ & $\mu_{\text {source }}=0$ & $\mu_{\text {source }}>0$ & $\mu_{\text {source }}=0$ \\
\hline & $\nabla \varphi=0$ & $\nabla \varphi>0$ & $\nabla \varphi>0$ & $\nabla \varphi=0$ \\
\hline & $d=0$ & $d=(3 / 2) B \nabla \varphi$ & $d>0\left(\mu \geq \mu_{\text {source }}\right)$ & $d>0$ \\
\hline & $j_{A}=0$ & $j_{A}=0$ & $j_{A}=0$ & $j_{A}=(3 / 2) B \mu$ \\
\hline & & & $0 \leq n_{s} \lesssim 0.3$ & \\
\hline Preferred at & none & Low $\mu$, low $T$ & High $\mu$, low $T$ & High $T$ \\
\hline $\begin{array}{l}\text { Important } \\
\text { properties }\end{array}$ & & & $\begin{array}{c}\text { Configuration } \mathbf{A}, \mathbf{B} \\
\text { merging at high } T \\
\text { and/or high } B\end{array}$ & \\
\hline
\end{tabular}

\section{Multiquark States and the Holographic Models}

In addition to baryons and mesons, the possibility of multiquark states were recognized by Gell-Mann since the proposal of the quark model. $Q^{m} \bar{Q}^{n}$-multiquark $(n+m>3)$ such as the tetraquark and dibaryon were proposed since 1977 by Jaffe [15-17] using the MIT bag model. There are theoretical models of colour-singlet multiquarks using interactions of various origins, for example, chromomagnetism, flux tube confinement, and hadronic molecules. Despite the theoretical possibilities, conclusive discovery of the multiquarks has yet to be confirmed experimentally (see [18] and references therein).

Series of experimental results from RHIC suggests that the produced QGP is strongly coupled (sQGP) [19-22]. The fact that the QGP is strongly coupled near-and-above the deconfinement temperature $T_{c}$ suggests the possibility of the existence of exotic bound states with colour degrees of freedom in the deconfined QGP. Recall that an interaction between two heavy quarks in the confined phase at $0<T<T_{c}$ can be described empirically by the screened Cornell potential

$$
V_{Q \bar{Q}}(r, T)=\sigma r\left[\frac{1-e^{-M_{D}(T) r}}{M_{D}(T) r}\right]-\frac{\alpha}{r}\left[e^{-M_{D}(T) r}\right]
$$

where $M_{D}$ is the Debye screening mass depending on $T$ and $\alpha$ is the effective coupling. The first part represents the (colour-screened) confining force due to QCD string with the effective string tension $\sigma$; it is around $0.20(\mathrm{GeV})^{2}$ as suggested by the lattice studies. The second part represents the effective (colour-screened) Coulomb potential due to transverse string oscillation. By definition, the effective string tension $\sigma$ vanishes at $T>T_{c}$. As a result, only the screened Coulomb part contributes to the interaction between quarks but within the range of screening length $M_{D}^{-1}$. Yet, as suggested by [23], a short string-like configuration of 
colour fields at low $T$ becomes longer strings at-and-near $T_{c}$ which contribute to the binding between quarks and gluons. Therefore, the bound states of gluons and quarks can exist in both colour-singlet and colour-nonsinglet forms in the sQGP.

The studies of the multibody bound states in the sQGP were initiated by Shuryak and colleagues [23-25]. Based on the studies in [23], three proposed multibody bound states: (i) diquark or "polymer-chain" ( $\bar{q} g g \cdots g q$ ); (ii) baryons (qqq); (iii) closed (3-)chains of gluons ( $g g g$ ) seem to exist only for $T=(1-1.5) T_{c}$. Importantly, the existence of these bound states could affect the thermodynamical and hydrodynamical properties of the sQGP.

The holographic models of colour-singlet baryon was originally investigated by Witten et al. $[9,10]$. In the $\mathrm{AdS}_{5} \times S^{5}$ background, a D5-brane wrapping the subspace $S^{5}$ with $N_{c}$ strings attached is proposed to be a dual description of a baryon. A holographic dual of a $k$-quark $\left(k<N_{c}\right)$ with colour degrees of freedom is discussed in [11] (see also [26]) for the supersymmetric background. There is a number of interesting articles investigating various possibilities of the multiquarks in both confined and deconfined medium, some of them consider deformed baryon vertex [12, 27-33]. Notably, [12] uses a simplified configuration with only one point-like baryon vertex to describe a variety of classes of the multiquarks with and without the colour degrees of freedom. We will focus our attention to such multiquark model in this paper.

In recent years, the AdS/CFT correspondence has attracted interests in its applicability to the phenomenological studies of non-perturbative QCD. However, this correspondence cannot provide the gravity dual of the large $N_{c}$ QCD. As its name suggests, the AdS/CFT have the gauge theory side, which is conformal, differing from the confining behaviour of the real-world QCD. There has been many attempts to engineer the holographic model whose the confining feature is taken into account $[8,34-36]$.

One natural way is to consider a stack of $N_{c}$ D4-branes, in Type IIA string theory, whose the world volume possesses one compact spatial direction [8]. In the near-horizon metric of a near-extremal D4-brane, the compactified spatial circle shrinks to zero size at some finite value of the radial direction representing a smooth cutoff of the spacetime. This feature can provide us with the confining spacetime background in which the potential between a holographic quark-antiquark bound state is mainly contributed by the tension of string lying along the "hard-wall." Consequently, the potential is linearly proportional to the separation between two ends of the string resulting in the confinement of quarks and antiquarks in the dual gauge theory.

At finite temperature, the time coordinate becomes Wick-rotated, and the asymptotic circumference of the time circle equals to the inverse of the temperature, $T^{-1}$. Consequently, the confining spacetime background at finite temperature has two compact directions. The metric of the geometry then can be written as

$$
d s^{2}=\left(\frac{u}{R_{\mathrm{D} 4}}\right)^{3 / 2}\left[\delta_{i j} d x^{i} d x^{j}+d \theta_{1}^{2}+f(u) d \theta_{2}^{2}\right]+\left(\frac{R_{\mathrm{D} 4}}{u}\right)^{3 / 2}\left[\frac{d u^{2}}{f(u)}+u^{2} d \Omega_{4}\right],
$$

where $\theta_{1}$ is the Euclidean time with temperature dependent period $\delta \theta_{1}=\beta \equiv T^{-1}, \theta_{2}$ is the compact spatial circle with period $\delta \theta_{2} \equiv(4 \pi / 3)\left(R^{3 / 2} / u_{\Lambda}^{1 / 2}\right)$, and $f(u) \equiv 1-\left(u_{\Lambda} / u\right)^{3}$. Notice that $f(u)$ equals zero for $u=u_{\Lambda}$ but equals one as $u$ approaches infinity. This $f(u)$ factor renders the $\theta_{2}-u$ subspace a cigar-like shape, while the $\theta_{1}-u$ subspace has a cylindrical shape. However, there is an alternative supergravity solution whose the time and the compact spatial coordinates exchange the role. That is, $\theta_{1}$ is the compact spatial coordinate with fixed 
Table 2: Brane configuration of the Sakai-Sugimoto model.

\begin{tabular}{lllllllllll}
\hline & 0 & 1 & 2 & 3 & 4 & 5 & 6 & 7 & 8 & 9 \\
\hline$N_{c} \mathrm{D} 4$ & $\mathrm{o}$ & $\mathrm{x}$ & $\mathrm{x}$ & $\mathrm{x}$ & $\mathrm{o}$ & & & & & \\
$N_{f} \mathrm{D} 8(\overline{\mathrm{D}} 8)$ & $\mathrm{o}$ & $\mathrm{x}$ & $\mathrm{x}$ & $\mathrm{x}$ & & $\mathrm{x}$ & $\mathrm{x}$ & $\mathrm{x}$ & $\mathrm{x}$ & $\mathrm{x}$ \\
\hline
\end{tabular}

circumference, $\theta_{2}$ is the Euclidean time with period $\delta \theta_{2}=\beta=(4 \pi / 3)\left(R^{3 / 2} / u_{T}^{1 / 2}\right)$, and $f(u) \equiv 1-\left(u_{T} / u\right)^{3}$. In other words, there are two geometries which can be the supergravity solution. The comparison of the free energy between these two competing geometries tells us about the deconfinement phase transition in the gauge theory side. It is important to emphasize that the asymptotic circumference of the time circle can be variable depending on the temperature, namely, $\delta \theta_{1}=T^{-1}$, while the $\theta_{2}$-circle has a fixed circumference. As a result, the phase transition occurs once the asymptotic circumferences of the two circles become the same in both geometries such that they have the same value of free energy. This gives rise to the deconfinement transition line in the $T-\mu$ phase diagram of the holographic nuclear matter $[8,37]$. For a concise review, see [38].

\subsection{The Sakai-Sugimoto Model}

More realistic holographic dual of the large $N_{c}$ QCD is the Sakai-Sugimoto (SS) model $[13,14]$. The brane construction of the SS model is a stack of $N_{c}$ D4 branes intersecting with $N_{f}$ D8- and $N_{f}$ anti-D8-branes, where $N_{f} \ll N_{c}$ such that the presence of the probe branes D8/anti-D8-branes does not affect the D4 background. This is called the probe limit, corresponding to the quenched approximation in the lattice QCD.

Stack of $N_{f}$ D8 and $\overline{D 8}$ branes are introduced as the flavour branes. They are located at separation distance $L_{0}$ along the compactified $x_{4}$ direction at the boundary $u \rightarrow \infty$. Openstring excitation with one end on the flavour branes behave like a chiral "quark." In the setup where D8 and $\overline{\mathrm{D} 8}$ are parallel in the $\left(x_{4}, u\right)$ projection, each chiral excitation on each stack of branes transform independently, therefore the theory has a chiral symmetry. For the setup where $\mathrm{D} 8$ and $\overline{\mathrm{D} 8}$ connect, forming a U-shape or a V-shape configuration in the $\left(x_{4}, u\right)$ projection, chiral symmetry is broken.

To obtain a SUSY broken QCD at low energy, the boundary conditions of the superpartners in the $x_{4}$ direction are chosen so that the zeroth modes vanish (Scherk-Schwarz mechanism). For energies below the first KK modes, the gauge theory therefore contains only gluons and chiral quarks. If the number of the stack of D4-branes source $N_{c}$ is chosen to be 3 , this low-energy gauge theory will look exactly like QCD. The brane configuration of the SS model is shown in Table 2.

Note that the " $x$ " sign signifies that the coordinate is occupied by an infinite extending direction of the D-brane world volume and the " $\mathrm{o}$ " sign means that the coordinate is occupied by a compact direction of the D-brane world volume. This holographic model is a QCD-like theory in many aspects. (i) It is nonsupersymmetric resulting from the antiperiodicity for superpartners around the $x^{4}$ circle. (ii) It has the confining behaviour and the deconfinement phase transition in the same way as mentioned above. In the confined phase, the $x^{4}$ coordinate is the cigar-like compact direction and $x^{0}$ (the Euclidean time) is the cylindrical compact direction. In the deconfined phase, the two coordinates exchange their roles. To summarize, the coordinates $\theta_{1}$ and $\theta_{2}$ in (2.2) can be specified in the confined and deconfined phase as shown in Table 3. 
Table 3: Geometric assignment of the compactified bulk coordinates in the Sakai-Sugimoto model.

\begin{tabular}{lcc}
\hline & Confined phase $\left(T<T_{\text {deconf }}\right)$ & Deconfined phase $\left(T>T_{\text {deconf }}\right)$ \\
\hline$\theta_{1}$ & $x^{0}$ & $x^{4}$ \\
$\theta_{2}$ & $x^{4}$ & $x^{0}$ \\
$f(u)$ & $1-\left(u_{\Lambda} / u\right)^{3}$ & $1-\left(u_{T} / u\right)^{3}$ \\
\hline
\end{tabular}

(iii) It has dynamical quarks resulting from the presence of the flavour branes. (iv) The phases of chiral symmetry breaking and chiral symmetric quark-gluon plasma ( $\chi \mathrm{S}-\mathrm{QGP}$ ) can be realized. There exist two configurations of the flavour D8- and anti-D8-branes, both satisfy the equation of motion. One is the connected configuration of the D8- and anti-D8-branes representing the chiral symmetry breaking phase. Another is the parallel configuration of the D8- and anti-D8-branes lying along the radial direction of the bulk spacetime representing the chiral symmetric phase. Note that $T_{\text {chiral }}=T_{\text {deconf }}$ when the separation between the D8and anti-D8-branes $L_{0} \gtrsim 0.97 R ; R \equiv$ the radius of the $x_{4}$ circle, while $T_{\text {deconf }}<T_{\text {chiral }}$ when $L_{0} \lesssim 0.97 R[37]$.

Since the SS model is the holographic model which gives exactly the particle content of the QCD at low energy, we will consider the holographic multiquarks in the deconfined SS model. The idea is to construct a gravity dual of the 5-dimensional gauge theory with chiral fermions which gives approximately the 4-dimensional QCD at low energy. The inevitable supersymmetry of the dual gauge theory in the string construction is broken at the position of the flavour branes used to introduce the chiral fermions. To construct the SS model, stack of D4-branes is used as the source to generate a curved background of the type IIA string theory. After taking the near-horizon limit and adding a black hole horizon, we arrive at the following background metric:

$$
\begin{gathered}
d s^{2}=\left(\frac{u}{R_{\mathrm{D} 4}}\right)^{3 / 2}\left(f(u) d t^{2}+\delta_{i j} d x^{i} d x^{j}+d x_{4}{ }^{2}\right)+\left(\frac{R_{\mathrm{D} 4}}{u}\right)^{3 / 2}\left(u^{2} d \Omega_{4}^{2}+\frac{d u^{2}}{f(u)}\right) \\
F_{(4)}=\frac{2 \pi N_{c}}{V_{4}} \epsilon_{4}, \quad e^{\phi}=g_{s}\left(\frac{u}{R_{\mathrm{D} 4}}\right)^{3 / 4}, \quad R_{\mathrm{D} 4}^{3} \equiv \pi g_{s} N_{c} l_{s}^{3}
\end{gathered}
$$

where $f(u) \equiv 1-u_{T}^{3} / u^{3}, u_{T}=16 \pi^{2} R_{\mathrm{D} 4}^{3} T^{2} / 9$. Note that the compact $x_{4}$ coordinate $\left(x_{4}\right.$ transverse to the probe D8-branes), with arbitrary periodicity $2 \pi R$, never shrinks to zero. The volume of the unit four-sphere $\Omega_{4}$ is denoted by $V_{4}$ and the corresponding volume 4 -form by $\epsilon_{4} . F_{(4)}$ is the 4 -form field strength, $l_{s}$ is the string length, and $g_{s}$ is the string coupling. The dilaton in this background has $u$-dependence, and its value changes along the radial direction $u$. This is a crucial difference in comparison to the AdS-Schwarzschild metric case where dilaton contribution is constant.

In the Sakai-Sugimoto model of D4-D8-branes construction, the D4-brane wrapping the $S^{4}$ is used as the baryon vertex. Remarkably, it was found that the baryon can also be realized as an instanton in the bulk of $N_{c}$ D4-brane-induced background spacetime, corresponding to baryon in the Skyrme model on the gauge theory side. This instanton can be described in terms of the Chern-Simons action in the bulk. Therefore, these two pictures of baryon are equivalent.

A Dn-brane wrapping an internal subspace $S^{n}$ accommodate a $U(1)$ which will couple to certain $n$-form field of the string background and becomes charged under the $U(1)$. To 


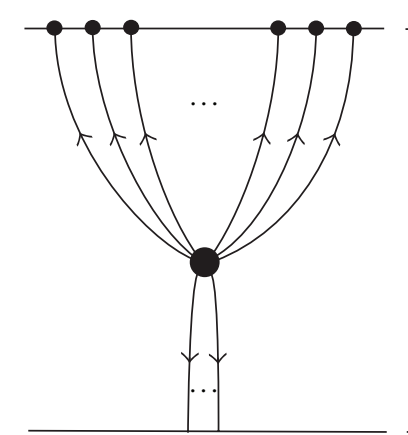

(a)

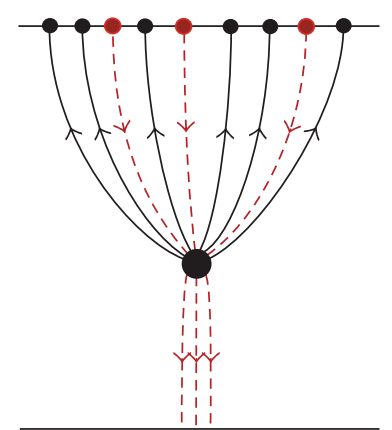

(b)

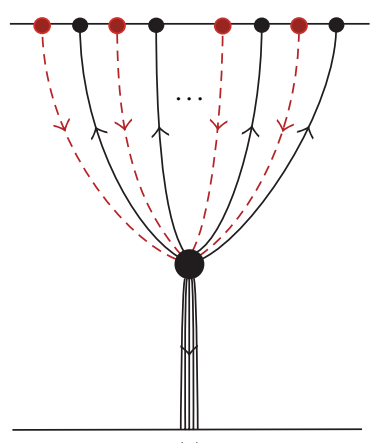

(c)

Figure 1: An illustration of the holographic multiquark states (a) $k$-baryon with $k_{h}=k<N_{c}$ and $k_{r}=$ $N_{c}-k,(b)\left(N_{c}+\bar{k}\right)$-baryon with $k_{h}=N_{c}+\bar{k}$ and $k_{r}=\bar{k}$, and (c) j-mesonance with $k_{h}=2 j$ and $k_{r}=N_{c}$.

cancel this charge for the entire background, a number of $N_{c}$ strings emerging from the vertex is required. While a string emerging from the vertex contributes a negative $U(1)$ charge, a string entering the vertex in the opposite orientation contributes a positive unit of $U(1)$ charge. Therefore, as long as the number of strings emerging from the vertex subtracting the number of strings entering the vertex is $N_{c}$, the configuration is allowed to exist since the total charge of the background is still zero.

Based on the charge cancelation at the vertex, three classes of exotic multiquarks are proposed in [12]. Namely, they are $k$-baryons, $\left(N_{c}+\bar{k}\right)$-baryon, and $j$-mesonance (strongly coupled bunch of mesons), corresponding to diquark, some exotic baryons such as pentaquark and a bunch of mesons, respectively. We parameterize $k_{h}$ as the number of hanging strings which extends from the vertex to the boundary and $k_{r}$ as the number of radial strings extending from the vertex to the horizon. Figure 1 shows 3 classes of the holographic multiquarks. Their conditions are summarized as the following.

For $k$-baryon,

$$
k_{h}+k_{r}=N_{c}, \quad k_{h}=k
$$

For $\left(N_{c}+\bar{k}\right)$-baryon,

$$
k_{h}-k_{r}=N_{c}, \quad k_{h}=N_{c}+\bar{k}
$$

For $j$-mesonance,

$$
k_{h}=2 j, \quad k_{r}=N_{c} .
$$

Note that the values of $\bar{k}$ and $j$ can be as large as $N_{c} \times N_{f}$.

\subsection{Force Balance Condition}

Using the field background shown in the last section, the total action of these exotic multiquark states can be generally written as

$$
S=S_{\mathrm{D} 4}+k_{h} S_{\mathrm{F} 1}+k_{r} \widetilde{S}_{\mathrm{F} 1}
$$


The DBI action of D4-brane

$$
S_{\mathrm{DBI}}=\int d x^{0} d \xi^{p} T_{p}, \quad T_{p}=\left(e^{-\phi}(2 \pi)^{p} \alpha^{\prime(p+1) / 2}\right)^{-1} \sqrt{-\operatorname{det}(g)}
$$

and the Nambu-Goto action of $k_{h}$ hanging strings and $k_{r}$ radial strings can be written as

$$
S_{\mathrm{D} 4}=\frac{\tau N_{c} u_{c} \sqrt{f\left(u_{c}\right)}}{6 \pi \alpha^{\prime}}, \quad S_{\mathrm{F} 1}=\frac{\tau}{2 \pi \alpha^{\prime}} \int_{0}^{L} d \sigma \sqrt{u^{\prime 2}+f(u)\left(\frac{u}{R}\right)^{3}}, \quad \widetilde{S}_{\mathrm{F} 1}=\frac{\tau}{2 \pi \alpha^{\prime}}\left(u_{c}-u_{\mathrm{T}}\right),
$$

respectively. Note that $\tau$ is the total time over which we evaluate the action and $u_{c}$ is the position where the D4-brane vertex is located.

Now let us write the force condition. As will be seen later, this is the equilibrium condition for the existence of the multiquark states. Assume the vertex to be a point at the cusp position $u_{c}$ that does not receive any distortion from the attached strings. The distortion of the baryon vertex due to the attached strings is discussed in details in $[39,40]$. Because of the spherical symmetry of the configuration in the $\left(x_{1}, x_{2}, x_{3}\right)$ subspace, the action is sensitive to only the variation in the holographic direction $u$. The variation of the action gives the volume term as well as the surface term. The equation of motion is obtained by requiring that the volume term and surface term vanishes separately. The volume term gives the EulerLagrange equation which determines the shape of the hanging strings. On the other hand, the surface term provides the equilibrium condition of the configuration at the tip $u_{c}$ under the variation in the $u$ direction, that is, the force balance condition at the cusp. It can be written as [12]

$$
\frac{N_{c}}{3} G_{0}(x)-k_{h} B+k_{r}=0
$$

where

$$
G_{0}(x) \equiv \frac{1+x^{3} / 2}{\sqrt{1-x^{3}}}, \quad x \equiv \frac{u_{T}}{u_{c}}<1, \quad B \equiv \frac{u_{c}^{\prime}}{\sqrt{{u_{c}^{\prime}}^{2}+f\left(u_{c}\right)\left(u_{c} / R_{\mathrm{D} 4}\right)^{3}}}
$$

Obviously, $B$ is always less than one, thus we obtain the equilibrium condition

$$
k_{h}>\frac{N_{c}}{3} G_{0}(x)+k_{r}
$$

Together with (2.5), (2.6), and (2.7), we obtain the lower bound of the hanging string parameter for each multiquark configuration as

for $k$-baryon,

$$
k_{h}=k>\frac{N_{c}}{6}\left(G_{0}(x)+3\right),
$$

$$
\text { for }\left(N_{c}+\bar{k}\right) \text {-baryon, }
$$

$$
k_{h}=N_{c}+\bar{k}>\frac{N_{c}}{3} G_{0}(x)+\bar{k}
$$


for $j$-mesonance,

$$
j>\frac{N_{c}}{6}\left(G_{0}(x)+3\right) .
$$

Note that $G_{0}(x)=1$ at $T=0$ and it is an increasing function of the temperature.

\subsection{Binding Energy and Screening Length}

Theoretically, all of these bound states are allowed to exist. But a question arises which multiquark state is more stable than another. This can be addressed by considering the binding energies of each class of the multiquarks. Naturally, the binding energy of each of these holographic bound states is the total energy of the configuration subtracted by the energy of the free quarks. Similar to the calculation of Wilson loop in [41], the binding energy in the large $N_{c}$ limit could be estimated to be the total classical action divided by $\tau$.

The solution or the shape of the hanging strings can be obtained by using the NambuGoto action from (2.10), the regulated energy of the hanging strings (subtracted by energy of the free quarks) is

$$
E_{\mathrm{F} 1}=\frac{1}{2 \pi} \int_{0}^{L} d \sigma \sqrt{u^{\prime 2}+\left(\frac{u}{R_{\mathrm{D} 4}}\right)^{3} f(u)}-\frac{1}{2 \pi} \int_{u_{T}}^{\infty} d u .
$$

From the equilibrium condition corresponding to the surface term, that is, (2.11) and (2.12), we obtain

$$
u_{c}^{\prime 2}=\frac{f\left(u_{c}\right) B^{2}}{1-B^{2}}\left(\frac{u_{c}}{R_{\mathrm{D} 4}}\right)^{3}
$$

where

$$
B=B\left(k_{h}, k_{r}, x\right)=\frac{N_{c}}{3 k_{h}} G_{0}(x)+\frac{k_{r}}{k_{h}} .
$$

Consider $S_{\mathrm{F} 1}$ in (2.10), the Lagrangian,

$$
\mathcal{L}=\sqrt{u^{\prime 2}+f(u)\left(\frac{u}{R}\right)^{3}}
$$

does not explicitly depend on $\sigma$, such that we can define the constant of motion

$$
\mathscr{\ell} \equiv \mathcal{L}-u^{\prime} \frac{\partial \mathcal{L}}{\partial u^{\prime}}=\text { const. }
$$

This leads to

$$
\frac{f\left(u_{c}\right)\left(u_{c} / R_{\mathrm{D} 4}\right)^{3}}{\sqrt{u_{c}^{\prime 2}+f\left(u_{c}\right)\left(u_{c} / R_{\mathrm{D} 4}\right)^{3}}}=\frac{f(u)\left(u / R_{\mathrm{D} 4}\right)^{3}}{\sqrt{u^{\prime 2}+f(u)\left(u / R_{\mathrm{D} 4}\right)^{3}}} .
$$

Eliminating $u_{c}^{\prime}$ using (2.18) and (2.22), we have the relation

$$
u^{\prime 2}=\frac{f(u)^{2}\left(u / R_{\mathrm{D} 4}\right)^{6}}{f\left(u_{c}\right)\left(u_{c} / R_{\mathrm{D} 4}\right)^{3}\left(1-B^{2}\right)}-f(u)\left(u / R_{\mathrm{D} 4}\right)^{3} .
$$


Using the above equation, we obtain the size of the radius of the multiquark state as

$$
L=\frac{R_{\mathrm{D}}^{3 / 2}}{u_{c}^{1 / 2}} \int_{1}^{\infty} d y \sqrt{\frac{\left(1-x^{3}\right)\left(1-B^{2}\right)}{\left(y^{3}-x^{3}\right)\left(y^{3}-x^{3}-\left(1-x^{3}\right)\left(1-B^{2}\right)\right)}},
$$

and together with (2.17), we also have

$$
E_{\mathrm{F} 1}=\frac{u_{c}}{2 \pi}\left\{\int_{1}^{\infty} d y\left[\sqrt{\frac{y^{3}-x^{3}}{\left(y^{3}-x^{3}\right)-\left(1-x^{3}\right)\left(1-B^{2}\right)}}-1\right]-(1-x)\right\}
$$

Therefore, the total energy of the vertex D-brane and the radial strings are

$$
\begin{aligned}
E & =\frac{N_{c} u_{T}}{2 \pi}\left(\frac{\sqrt{1-x^{3}}}{3 x}+\left(\frac{k_{h}}{N_{c}}\right) \frac{\varepsilon}{x}+\left(\frac{k_{r}}{N_{c}}\right) \frac{1-x}{x}\right) \\
& \sim \frac{N_{c}^{2}}{L^{2}}
\end{aligned}
$$

where $\varepsilon$ represents the terms within the brace of (2.25).

By numerical calculations, we compare the $E / N_{c}$ (the energy per degrees of freedom) versus $L$ (the size of radius of the bound states) of the 3 classes of the multiquark as in Figure 2. The deeper the binding energy is, the harder the multiquark will melt in the thermal bath. From Figure 2, the colour singlet $N_{c}$-baryon has deeper binding well than the $\left(k<N_{c}\right)$-baryon and $\left(N_{c}+\bar{k}\right)$-baryon. As expected, the $\left(k<N_{c}\right)$-multiquark is bound more tightly as $k$ gets larger. For $\left(N_{c}+\bar{k}\right)$-baryon, the bound state is less tightly bound, as $\bar{k}$ increases. Similarly, a $j$-mesonance has the binding energy less than $j$ mesonic states. It becomes closer to $j$ mesons as $j$ grows.

The screening length $L^{*}$ can also be numerically calculated. It is defined to be the value at which the binding energies become zero from negative values at small distances. The numerical results, as shown in Figure 3, indicate that the multiquark states of all classes have smaller screening lengths as the temperature increases, with approximately $L^{*} \sim 1 / T$ for a fixed $k, \bar{k}$, and $j$. Furthermore, $L^{*}$ is larger as $k$ and $j$ increases for $k$-baryon and $j$-mesonance, respectively, while it is smaller as $\bar{k}$ increases for $\left(N_{c}+\bar{k}\right)$-baryon. Interestingly, the saturation

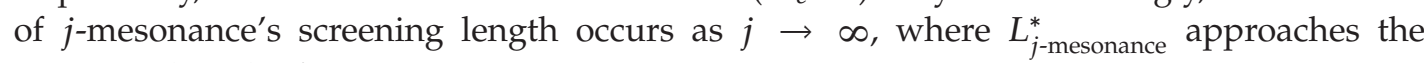
screening length of a meson $L_{\text {meson }}^{*}$.

\section{Thermodynamic Properties of Holographic Multiquark}

In the non-antipodal SS model, the holographic plasma can have two distinctive phase transitions; a deconfinement and the chiral symmetry restoration [37]. The deconfinement could occur at lower temperature than the chiral symmetry restoration. For the temperature in between the two transitions, quarks and gluons are deconfined from the confining flux tube but still interact strongly among each other through the remaining screened Coulomb-type $S U\left(N_{c}\right)$ potential. Therefore, it is possible to have the multiquark phase in the temperature 


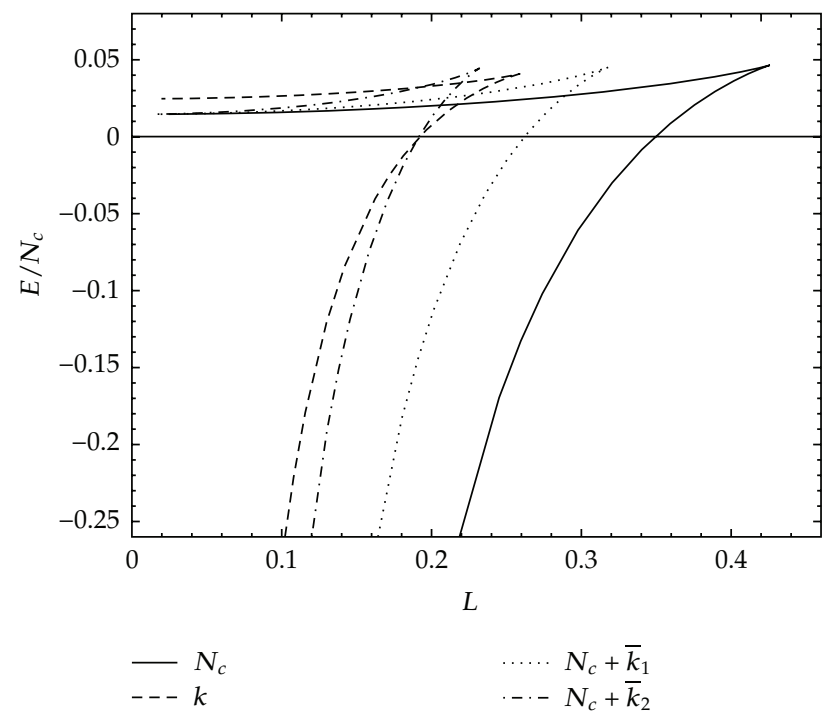

Figure 2: Comparison of the potential per $N_{c}$ between $N_{c}$-baryon, $k$-baryon, and $\left(N_{c}+\bar{k}\right)$-baryon for $k / N_{c}=0.8, \overline{k_{1}} / N_{c}=2 / 3$, and $\overline{k_{2}} / N_{c}=2$ at temperature $T=0.25$.

range between that of the deconfinement and the chiral phase transition. This is consistent, at least in a qualitative way, with the studies of the multibody bound states in the sQCD in the framework of the real QCD [23] mentioned in the previous section.

To actually understand the physics of deconfined QGP, it is thus crucial to investigate the thermodynamical properties of the holographic multiquark phase. In order to extract the thermodynamic potential from the gravity dual model, the path integral approach in quantum gravity [42] has been used. In this technique, the time direction is circled with period $\beta=1 / T$ in the same manner as the thermal circle in the finite temperature quantum field theory. As discussed in [43] based on the early works [44, 45], the grand canonical potential, or the Gibbs free energy, $\Omega(T, \mu)$ has the leading contribution from the classical Euclidean action of the bulk theory in the grand canonical ensemble, that is, $\Omega(T, \mu) \sim S_{\text {bulk }}^{\text {on-shell }}$. Similarly, the Helmholtz free energy $F\left(T, n_{b}\right)$ has the leading contribution from the Legendre transform with respect to the baryonic charge of the classical Euclidean action, that is, $F\left(T, n_{b}\right) \sim \widetilde{S}_{\text {bulk }}^{\text {on-shell }}$ in the canonical ensemble. If we are interested in the situation of nonfixed baryon number density but fixed chemical potential, the relevant thermodynamic potential is the grand canonical potential.

The deconfinement phase transition can be realized as the Hawking-Page transition due to the competition between the action of the background geometry corresponding to the confined phase and the action of the background corresponding to the deconfined phase [8]. Since the coloured multiquark matter can exist only in the deconfined phase (however, the colour-singlet multiquarks such as a baryon can also exist in the confined phase), its grand canonical potential in the Sakai-Sugimoto model is $\beta$ times the combination of the classical action of the deconfining spacetime geometry and the configuration of flavour sector, which includes $N_{f}$ D8- $\overline{\mathrm{D} 8}$-branes, the probe D4-brane vertex, and the radial strings. Note that the part of hanging strings, extending from the baryon vertex to the flavour branes, is neglected, and we assume that there is no distortion of the vertex due to the connecting strings (such distortion is discussed in $[39,40])$. As a result, the baryon vertex is embedded into the flavour branes and becomes an instanton on them. 


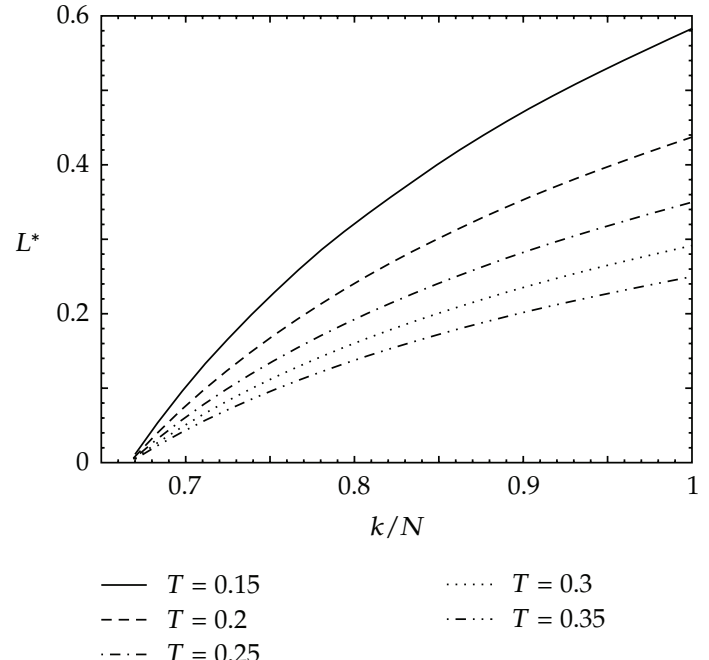

(a)

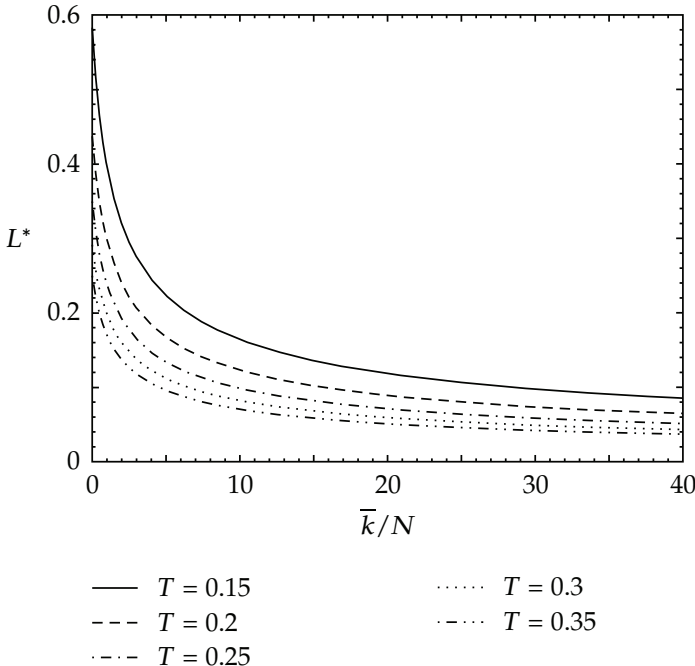

(b)

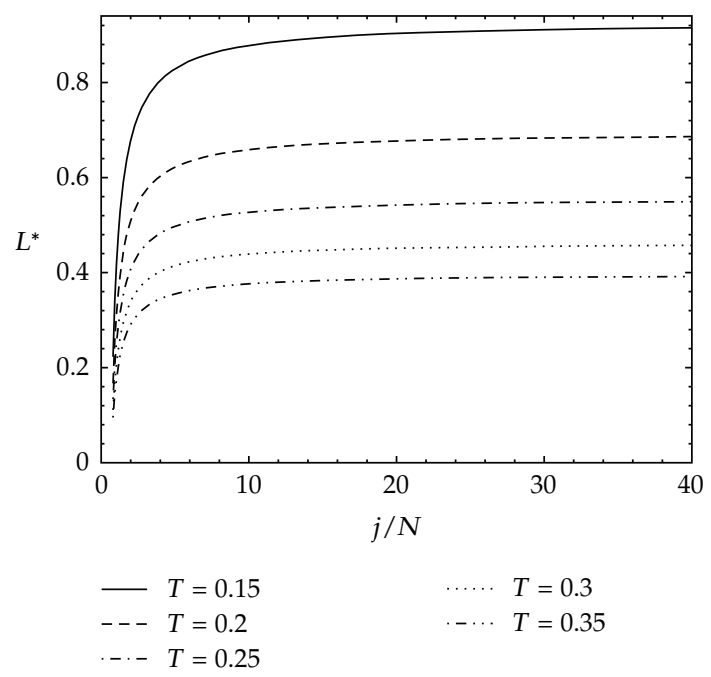

(c)

Figure 3: (a) the screening lengths of $k$-baryons with respect to $k$, (b) the screening lengths of $\left(N_{c}+\bar{k}\right)$ baryons with respect to $\bar{k}$, and (c) the screening lengths of $j$-mesonance with respect to $j$ for $T=0.15-0.35$.

Intriguingly, whereas the deconfining spacetime geometry action (scales as $N_{c}^{2}$ ) dominates the action of the fundamental matter sector (scales as $N_{c} N_{f}$ ), the dominating part can be ignored in the consideration of the holographic phase transition in the deconfined phase. Above the deconfinement, the multiquarks phase competes with the vacuum phase and the chiral-symmetric quark-gluon plasma. In this section, we will explore the phase diagram of the deconfined nuclear matter especially the region of the parameter space where the multiquark phase is dominant. Then we will study the thermodynamics of the multiquark nuclear matter in the dilute and dense limits. 

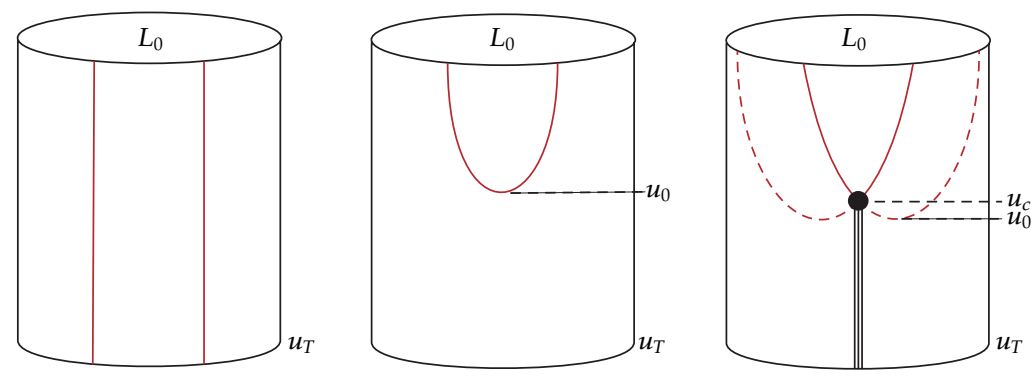

Figure 4: Configurations of $x^{S}$-QGP (a) vacuum (b), and multiquark nuclear phase (c) in $x_{4}-u$ projection.

\subsection{Phase Diagram}

In order to determine a phase diagram, we need to find which phase of nuclear matter is thermodynamically preferred to others in a particular region of the parameter space. For the grand canonical ensemble at a fixed $\mu$, the thermodynamically preferred phase is the configuration with the grand canonical potential smaller than that of all other phases.

We will first determine the brane configuration in the presence of the external sources by minimizing the classical action. The position of the tip $u_{c}$ of the $\mathrm{D} 8-\overline{\mathrm{D}} 8$ will be determined from the equilibrium condition at the tip. The resulting brane configuration corresponds to the multiquark nuclear phase. On the other hand, the vacuum phase corresponds to the configuration with zero sources and density, and the $x^{S}$-QGP phase is dual to the brane configuration with parallel branes without a tip. The $x_{4}-u$ projecion of the brane configuration for each deconfined phase is schematically shown in Figure 4. Then we will define the normalized grand canonical potential using the action of the D8-branes. The action of each brane configuration is divergent from the limit $u \rightarrow \infty$. By subtracting with the action of the vacuum configuration, we can regulate the grand canonical potential of each configuration. By comparing the grand canonical potential of each phase, we finally draw a phase diagram in $(\mu, T)$ parameter space.

Start with the DBI action of the D8-branes

$$
S_{\mathrm{D} 8}=-\mu_{8} \int d^{9} X e^{-\phi} \operatorname{Tr} \sqrt{-\operatorname{det}\left(g_{M N}+2 \pi \alpha^{\prime} \mathcal{F}_{M N}\right)},
$$

where $g_{M N}$ is the induced metric of the D8-world volume and the field strength tensor of the gauge group $U\left(N_{f}\right)$ living in the $N_{f}$ flavour branes is

$$
\mathcal{F}=d A+i A \wedge A
$$

While the full D8-brane world-volume gauge fields is

$$
\mathcal{A}=\mathcal{A}_{S U\left(N_{f}\right)}+\frac{1}{\sqrt{2 N_{f}}} \widehat{A},
$$


we turn on only the time component of the diagonal subgroup $U(1)$ part, $\hat{A} / \sqrt{2 N_{f}}$ in order to introduce the finite chemical potential, or equivalently finite baryon density [43, 46]. From the deconfining spacetime metric, (2.3), the DBI action of the D8-branes becomes

$$
S_{\mathrm{D} 8}=\mathcal{N} \int d u u^{4} \sqrt{f(u)\left(x_{4}^{\prime}(u)\right)^{2}+u^{-3}\left(1-\left(\widehat{a}_{0}^{\prime}(u)\right)^{2}\right)},
$$

where the factor $\mathcal{N}$ is defined to be

$$
\mathcal{N} \equiv \frac{\mu_{8} \beta N_{f} \Omega_{4} V_{3} R^{5}}{g_{s}}
$$

as the result of integrating out all world-volume coordinates except the holographic direction $u$. And $\hat{a}$ are defined as

$$
\widehat{a}=\frac{2 \pi \alpha^{\prime} \hat{A}}{R \sqrt{2 N_{f}}}
$$

The action does not depend on $\widehat{a}_{0}$, hence we can define a constant

$$
\begin{aligned}
d & \equiv \frac{\delta S_{\mathrm{D} 8}}{\delta \widehat{\boldsymbol{F}}_{0 u}} \\
& =\frac{u \widehat{a}_{0}^{\prime}(u)}{\sqrt{f(u)\left(x_{4}^{\prime}(u)\right)^{2}+u^{-3}\left(1-\left(\widehat{a}_{0}^{\prime}(u)\right)^{2}\right)}},
\end{aligned}
$$

which can be interpreted as the electric displacement field along the holographic direction. Similarly for the variation of action with respect to $x_{4}(u)$, we can define another constant of motion, says $\Gamma$, so that we can rearrange to obtain

$$
\left(x_{4}^{\prime}(u)\right)^{2}=\frac{1}{u^{3} f(u)}\left[\frac{f(u)\left(u^{8}+u^{3} d^{2}\right)}{\Gamma^{2}}-1\right]^{-1},
$$

from which at large $u$

$$
x_{4}(u) \approx \frac{L_{0}}{2}-\frac{2}{9} \frac{\Gamma}{u^{9 / 2}}
$$

where $L_{0}$ is the separation between $\mathrm{D} 8$ and $\overline{\mathrm{D} 8}$ branes at $u \rightarrow \infty$ defined by

$$
L_{0} \equiv 2 \int_{u_{c}}^{\infty} x_{4}^{\prime}(u) d u
$$

The parameter $\Gamma$ can be thought of as the curvature of the D8- $\overline{\mathrm{D} 8}$-branes around the cusp. It becomes zero when the flavour embedding is in the parallel configuration representing the chiral-symmetric QGP. According to [47], this means that it can be used as an order parameter of the nuclear matter $\mathrm{x}$ S-QGP phase transition. 
We will set $L_{0}=1$ to allow the possibility of the chiral symmetry restoration as separate phase transition from the deconfinement. Apply the equilibrium condition at the cusp $u_{c}$ (see the appendix), we obtain $\Gamma$ in terms of $x_{4}^{\prime}\left(u_{c}\right)$,

$$
\begin{aligned}
\Gamma & =\frac{f\left(u_{c}\right) \sqrt{u_{c}^{8}+u_{c}^{3} d^{2}}}{\sqrt{f\left(u_{c}\right)\left(x_{4}^{\prime}\left(u_{c}\right)\right)^{2}+u_{c}^{-3}}} x_{4}^{\prime}\left(u_{c}\right) \\
& =u_{c}^{3} f\left(u_{c}\right)\left[\left(u_{c}^{5}+d^{2}\right)-\frac{d^{2} \eta_{c}^{2}\left(T, n_{s}\right)}{9 f\left(u_{c}\right)}\right],
\end{aligned}
$$

where

$$
\eta_{c}\left(T, n_{s}\right) \equiv 1+\frac{1}{2}\left(\frac{u_{T}}{u_{c}}\right)^{3}+3 n_{s} \sqrt{f\left(u_{c}\right)}
$$

Note that the formula of $x_{4}^{\prime}\left(u_{c}\right)$ is derived from the variation of the total action, in which the D8-branes action has been transformed to possess the dynamical variable $d$ rather than $a_{0}^{\prime}$,

$$
\frac{\partial \widetilde{S}_{\text {total }}}{\partial u_{c}}=\frac{\partial\left(\widetilde{S}_{\mathrm{D} 8}+S_{\mathrm{D} 4}+\widetilde{S}_{\mathrm{F} 1}\right)}{\partial u_{c}}=0 .
$$

This is reminiscent of the way we obtain the equilibrium condition of the multiquark vertex, (2.11), minimizing the surface terms with respect to $u_{c}$.

It is important to emphasize that the parameter $n_{s}$ is the number of radial strings in the unit of $N_{c}$. Due to the zero length of the hanging strings, we cannot distinguish the different classes of multiquarks proposed in Section 2 for a particular value of $n_{s}$, but some possibilities, $j$-mesonance for example, can be ruled out by examining their thermodynamic stability. This will be shown in this subsection.

Before going further to calculate the classical action, let us comment about the electric displacement $d$. It has been shown in [48] that it is related to the baryon number density. The baryon number density corresponds to the number density of instantons, $n_{4}$, on the D8branes. It also contributes to the Chern-Simons (CS) action of the flavour branes [13].

Beginning with the D8-brane CS term [49]

$$
S_{\mathrm{D} 8}^{\mathrm{CS}}=\frac{\mu_{8}}{3 !} \int_{R^{4} \times R_{+} \times S^{4}} C_{3} \wedge \operatorname{Tr}\left(2 \pi \alpha^{\prime} \mathcal{F}\right)^{3} .
$$

It is convenient to rescale the RR (Ramond-Ramond) field following the Appendix of [13] such that

$$
\begin{aligned}
S_{\mathrm{D} 8}^{\mathrm{CS}} & =\frac{1}{48 \pi^{3}} \int_{R^{4} \times R_{+} \times S^{4}} C_{3} \wedge \operatorname{Tr} \mathcal{F}^{3} \\
& =\frac{1}{48 \pi^{3}} \int_{R^{4} \times R_{+} \times S^{4}} F_{4} \omega_{5}(\mathcal{A}),
\end{aligned}
$$


where the last expression is obtained through the integration by part. $F_{4}=d C_{3}$ is the RR 4 -form field strength and $\omega_{5}(\mathcal{A})$ is the CS 5-form:

$$
\omega_{5}(\mathcal{A})=\operatorname{Tr}\left(\mathcal{A}^{2}-\frac{1}{2} \mathscr{A}^{3} \mathcal{F}+\frac{1}{10} \mathscr{A}^{5}\right)
$$

satisfying $d \omega_{5}=\operatorname{Tr} \mathcal{F}^{3}$. Using the fact that integrating the $F_{4}$ flux over the $S^{4}$ in the $N_{c}$ D4branes background gives

$$
\frac{1}{2 \pi} \int_{S^{4}} F_{4}=N_{c}
$$

and the relevant term is only the first term in the CS 5-form, (3.18), once turning on only the time-component of the diagonal $U(1)_{V}$ field, we obtain

$$
S_{\mathrm{D} 8}^{\mathrm{CS}}=\frac{N_{c}}{24 \pi^{2}} \int_{R^{4} \times R_{+}} \frac{1}{\sqrt{2 N_{f}}} \widehat{A}_{0} \wedge \operatorname{Tr}(\mathcal{F} \wedge \mathcal{F}) .
$$

Assuming a uniform distribution of D4-branes in $\mathbb{R}^{3}$ at $u=u_{c}$, we have [50]

$$
\frac{1}{8 \pi^{2}} \operatorname{Tr}(\mathcal{F} \wedge \mathcal{F})=R^{-3} n_{4} \delta\left(u-u_{c}\right) d^{3} \mathbf{x} d u
$$

where $n_{4}$ is defined to be the (dimensionless) number density of instantons, or the wrapped D4-branes, at $u=u_{c}$. From the viewpoint that the low-energy effective theory on the D8-brane includes the Skyrme model [13], it is natural to interpret $n_{4}$ as the baryon number density.

Using (3.6), (3.20), and (3.21), we obtain

$$
S_{\mathrm{D} 8}^{\mathrm{CS}}=\frac{n_{4} N_{c} \beta V_{3}}{2 \pi \alpha^{\prime} R^{2}} \int_{u_{c}}^{\infty} d u \widehat{a}_{0}(u) \delta\left(u-u_{c}\right) .
$$

From both the DBI and CS parts of D8-branes action, the equation of motion with respect to the $U(1)$ gauge field gives [48]

$$
n_{4}=\frac{2 \pi \alpha^{\prime} R^{2} N}{\beta V_{3} N_{c}} d
$$

Note that this reflects the one-dimensional electrostatic effect in which the point electric charges are put at $u_{c}$, generating constant electric field in the holographic direction.

The normalized grand canonical potential from the holographic model can be defined using the D-brane action as

$$
\Omega(\mu)=\frac{1}{\mathcal{N}} S_{\mathrm{D} 8}\left[x_{4}(u), \widehat{a}_{0}(u)\right]_{c l}
$$

Since the D-brane action diverges from the limit $u \rightarrow \infty$ of the integration, the grand canonical potential needs to be regulated by subtracting with the grand canonical potential of the vacuum phase at the same temperature. 
Apart from the grand canonical potential, the chemical potential also needs to be holographically identified in the dual bulk theory. To this end, the time component of the $U(1)_{V}$ gauge field $\widehat{A}_{0}$ is taken into account. From the field/operator matching scheme, a bulk field evaluated at $u \rightarrow \infty$, that is, the boundary of the spacetime background plays a role as the source of the dual operator in the generating function of correlation functions in quantum field theory. In other words, this nonnormalizable mode of the bulk field is dual to the coefficient of the field operator. Since the chemical potential is the coefficient of the charge density operator term, it can be holographically identified as $\widehat{A}_{0}(\infty)$. By rescaling for convenience, we can write the dimensionless chemical potential as

$$
\mu=\widehat{a}_{0}(\infty)
$$

Similarly, the baryon number density in our normalization is given by

$$
n_{b}=-\frac{\partial \Omega(T, \mu)}{\partial \mu}=d
$$

even though the true baryon number density is $n_{4}$ defined in (3.23). Consequently, $d$ can then be used to denote the baryon number density.

Since the free energy in the canonical ensemble is the combination of the on-shell Legendre-transformed D8-brane action and the source term, it is convenient to obtain $\mu$ through

$$
\mu=\frac{\partial \mathscr{F}_{E}(T, d)}{\partial d}
$$

where the free energy is holographically defined as the Legendre-transformed D8-brane action plus the source terms

$$
\mathcal{F}_{E}(T, d)=\frac{1}{\mathcal{N}}\left(\widetilde{S}_{\mathrm{D} 8}\left[T, x_{4}(u), d(u)\right]_{\text {on-shell }}+S_{\text {source }}\left(T, d, u_{c}\right)\right) .
$$

The Legendre-transformed action $\widetilde{S}_{\mathrm{D} 8}$ is given by

$$
\begin{aligned}
\widetilde{S}_{\mathrm{D} 8} & =S_{\mathrm{D} 8}+\mathcal{N} \int_{u_{c}}^{\infty} d(u) \widehat{a}_{0}^{V^{\prime}} d u, \\
& =\mathcal{N} \int_{u_{c}}^{\infty} d u u^{4} \sqrt{f(u)\left(x_{4}^{\prime}(u)\right)^{2}+u^{-3}} \sqrt{1+\frac{d^{2}}{u^{5}}}
\end{aligned}
$$


The chemical potential can then be written as

$$
\begin{aligned}
\mu=\frac{1}{\mathcal{N}}\left\{\left.\int_{u_{c}}^{\infty} d u\left(\frac{\delta \tilde{S}_{\mathrm{D} 8}}{\delta d(u)}+\frac{\delta \tilde{S}_{\mathrm{D} 8}}{\delta x_{4}^{\prime}} \frac{\partial x_{4}^{\prime}}{\partial d}\right)\right|_{T, L_{0}, u_{c}} ^{\text {on-shell }}\right. \\
\left.\quad+\left.\left.\frac{\partial u_{c}}{\partial d}\right|_{T, L_{0}}\left(\frac{\partial \widetilde{S}_{\mathrm{D} 8}}{\partial u_{c}}+\frac{\partial S_{\text {source }}}{\partial u_{c}}\right)\right|_{d, T, L_{0}} ^{\text {on-shell }}+\left.\frac{\partial S_{\text {source }}}{\partial d}\right|_{T, L_{0}, u_{c}}\right\} .
\end{aligned}
$$

The second, third, and fourth terms drop out. It is clear from (3.15) corresponding to the equilibrium at the cusp that the third and fourth terms vanish. For the second term, it is because $\delta \widetilde{S}_{\mathrm{D} 8} / \delta x_{4}^{\prime}(u)$ is constant as can be seen from (3.30) that $\widetilde{S}_{\mathrm{D} 8}$ depends only on $x_{4}^{\prime}$. Integrating over the remaining gives $\partial L_{0} / \partial d$, which is zero, due to the scale fixing condition $L_{0}=1$. Hence we obtain

$$
\mu=\int_{u c}^{\infty} \widehat{a}_{0}^{\prime}(u)+\left.\frac{1}{\mathcal{N}} \frac{\partial S_{\text {source }}}{\partial d}\right|_{T, L_{0}, u_{c}} .
$$

Now, it is ready to express the grand canonical potential for the multiquark (baryon corresponds to $n_{s}=0$ ) phase. Using (3.24), (3.4), (3.8), (3.9), we obtain the formulae of the grand canonical potential for the multiquark matter. The chemical potential can be calculated from (3.32) by eliminating $a_{0}^{\prime}$ via (3.8) and substituting (3.9). The grand canonical potential and the baryon chemical potential of the phases can be expressed as the following:

\section{Nuclear (Including Exotics) Phase}

$$
\begin{gathered}
\Omega_{\mathrm{nuc}}=\int_{u_{c}}^{\infty} d u\left[1-\frac{\Gamma^{2}}{f(u)\left(u^{8}+u^{3} d^{2}\right)}\right]^{-1 / 2} \frac{u^{5}}{\sqrt{u^{5}+d^{2}}} \\
\mu_{\mathrm{nuc}}=\int_{u_{c}}^{\infty} d u\left[1-\frac{\Gamma^{2}}{f(u)\left(u^{8}+u^{3} d^{2}\right)}\right]^{-1 / 2} \frac{d}{\sqrt{u^{5}+d^{2}}}+\frac{1}{3} u_{c} \sqrt{f\left(u_{c}\right)}+n_{s}\left(u_{c}-u_{T}\right) .
\end{gathered}
$$

Recall that $\Gamma$ depends on $u_{c}, d, T$ and $n_{s}$ according to (3.12) and (3.14). The last two terms in (3.34) come from the derivative of the source-term action with respect to $d$.

There are at least other two phases that compete with the multiquark phase: the vacuum phase and the chiral-symmetric QGP phase. From the above formula of $\Omega_{\text {nuc }}$ and $\mu_{\text {nuc }}$, we can obtain the grand canonical potential and the chemical potential of the vacuum simply by (i) setting $d=0$, (ii) dropping the source terms in (3.34), (iii) changing the lower bound of integration from $u_{c}$ to $u_{0}$, and (iv) replacing $\Gamma$ by the constant of motion in the vacuum configuration, from $\delta S_{\mathrm{D} 8} / \delta x_{4}^{\prime}, \Gamma_{0}=f\left(u_{0}\right) u_{0}^{8}$. Thus we obtain

Vacuum Phase, $d=0$

$$
\Omega_{\mathrm{vac}}=\int_{u_{0}}^{\infty} d u\left[1-\frac{\Gamma_{0}^{2}}{f(u) u^{8}}\right]^{-1 / 2} u^{5 / 2}
$$


Similarly, the grand canonical potential and the chemical potential of the XS-QGP phase can be obtained by setting $x_{4}^{\prime}(u)=0$, reflecting its parallel configuration, and turning off the source terms. That is, setting $\Gamma=0$ in (3.33) and (3.34), changing lower bound of integration to $u_{T}$ and dropping the source terms in (3.34) give

XS-QGP Phase, $x_{4}^{\prime}(u)=0$

$$
\begin{aligned}
& \Omega_{\mathrm{qgp}}=\int_{u_{T}}^{\infty} d u \frac{u^{5}}{\sqrt{u^{5}+d^{2}}}, \\
& \mu_{\mathrm{qgp}}=\int_{u_{T}}^{\infty} d u \frac{d}{\sqrt{u^{5}+d^{2}}} .
\end{aligned}
$$

The phase transition in the parameter space $(\mu, T)$ is obtained by comparing the grand canonical potential between two phases at a particular $T$ and $\mu$. Let us say the transition between phase 1 with the grand canonical potential $\Omega_{1}$ and phase 2 with $\Omega_{2}$. Phase 1 is thermodynamically preferred once $\Omega_{1}<\Omega_{2}$ and vice versa. There is a first order phase transition when $\Omega_{1}=\Omega_{2}$. This kind of phase transition can be seen in the transition between the vacuum and $x \mathrm{~S}$-QGP phases and the transition between the $x \mathrm{~S}$-QGP and the nuclear matter. However, the phase transition between the vacuum and nuclear matter phases is of the second-order as seen from (3.34). The density $d$ is continuous near $\mu=\mu_{\text {onset, }}$ which is $\mu_{\text {nuc }}(d=0)$, and behaves as $d \sim\left(\mu_{\text {nuc }}-\mu_{\text {onset }}\right)$. Note that this reflects the absence of the interactions between the multiquarks and baryons. As a result, the critical chemical potential defined to be the value, at which

$$
\frac{\partial d}{\partial \mu}=-\frac{\partial^{2} \Omega}{\partial \mu^{2}}
$$

has a discontinuity, is given by $\mu_{\text {onset }}$. By numerical calculations, the phase transition lines can be obtained as shown in Figure 5. The phase diagram between the chiral-broken vacuum and the chiral symmetric QGP phases was first obtained in [51]. The phase diagram of all 3 deconfined phases including the baryonic nuclear phase (without the multiquarks) is originally discussed in [48].

This phase diagram also shows the presence of the multiquark phase which can be mixed in the region of normal baryon phase (with $n_{s}=0$ ), say $\mathrm{B}+\mathrm{C}$ for $n_{s}=0.1$, and $\mathrm{C}$ for $n_{s}=0.3$. The multiquark matter with $0<n_{s}<0.5$ is less stable than the normal baryon due to the larger value of the grand canonical potential. Above $n_{s}=0.3$, it can be shown that the multiquark phase is unstable to density fluctuations, that is, $\partial \mu / \partial d<0$, in some regions of high $T$ and certain range of $\mu$. For approximately $n_{s}>0.5$, the multiquark phase is unstable thermodynamically to density fluctuations for most of the temperatures.

If the multiquark matter can exist in the quark-gluon plasma, it should mix with the normal baryon states in thermal equilibrium with the populations following the Boltzmann factor

$$
\exp \left(-\frac{E}{k_{B} T}\right)
$$

where $E$ is the binding energies for the states. It is interesting to explore more about the population of these multiquark states in the quark-gluon plasma potentially produced in the 


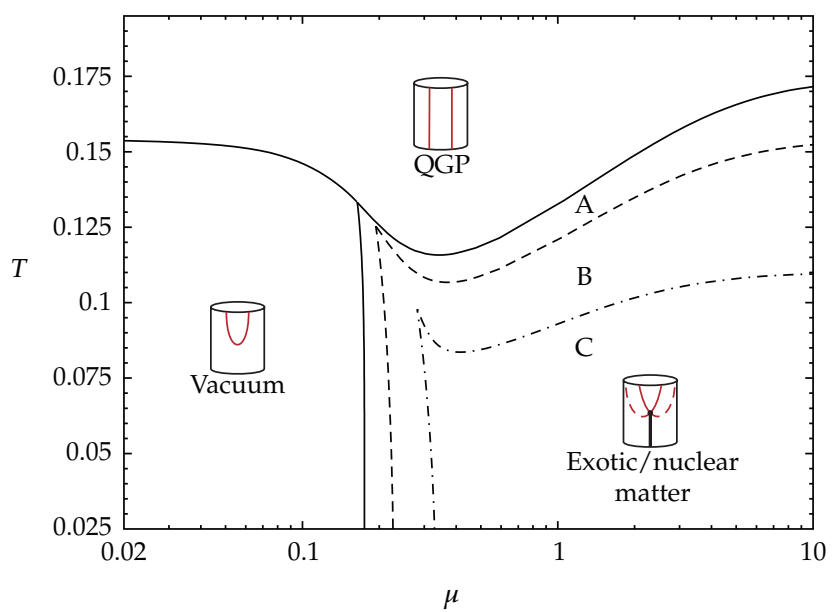

Figure 5: The phase diagram of deconfined nuclear matters in the Sakai-Sugimoto model. Multiquark phase is shown as the region on the lower right corner where it is divided into 3 parts according to the value of the colour strings $n_{s}$. A, B, C represent the region where multiquark phase with $n_{s}=0\left(N_{c^{-}}\right.$ baryon), $0.1,0.3$ is the most thermodynamically preferred.

heavy-ion collision experiments such as the RHIC and the LHC. Existence of these multiquark states contribute significantly to the hydrodynamical and thermodynamical properties of the deconfined plasma.

\subsection{Thermodynamic Relations}

In the grand canonical ensemble, the grand canonical potential $G_{\Omega}$ is the function of the dynamical variables: the volume $V$, the temperature $T$, and the chemical potential $\mu$. Its differential is

$$
d G_{\Omega}=-P d V-S d T-N d \mu,
$$

where the coefficients $P, S$, and $N$ are the pressure, entropy, and the total number of particles, respectively. It is better to understand the system of QGP in terms of volume density of extensive parameters. Let us define the volume density of $G_{\Omega}, S$, and $N$ to be $\Omega, s$, and $d$, respectively. Hence, the pressure is

$$
P=-\frac{G_{\Omega}}{V} \equiv-\Omega(T, \mu) .
$$

From (3.26) and (3.41), we use the chain rule to obtain

$$
\left.\frac{\partial P}{\partial d}\right|_{T}=\left.\frac{\partial \mu}{\partial d}\right|_{T} d
$$

so that

$$
P\left(d, T, n_{s}\right)=\mu\left(d, T, n_{s}\right) d-\int_{0}^{d} \mu\left(d^{\prime}, T, n_{s}\right) d\left(d^{\prime}\right),
$$

where we have assumed that the regulated pressure is zero when there is no nuclear matter, that is, $d=0$. 
While the equations of motion cannot be obtained analytically, we can find them in the limit of very small and very large density. Using (3.34) and (3.43), we take $d \approx 0$ and use the binomial expansion, then (see [52] for details)

$$
P \simeq \frac{\alpha_{0}}{2} d^{2}-\frac{3 \beta_{0}\left(n_{s}\right)}{4} d^{4}
$$

where

$$
\begin{aligned}
\alpha_{0} & \equiv \int_{u_{0}}^{\infty} d u \frac{u^{-5 / 2}}{1-f_{0} u_{0}^{8} / f u^{8}}, \\
\beta_{0}\left(n_{s}\right) & \equiv \int_{u_{0}}^{\infty} d u \frac{u^{-5 / 2}}{2 \sqrt{1-f_{0} u_{0}^{8} / f u^{8}}}\left(\frac{f_{0} u_{0}^{3}}{f u^{8}-f_{0} u_{0}^{8}}\left(1-\frac{\eta_{0}^{2}}{9 f_{0}}-\frac{u_{0}^{5}}{u^{5}}\right)+\frac{1}{u^{5}}\right) .
\end{aligned}
$$

Note that we have used the fact that $\eta_{c}$ of (3.14) becomes $\eta_{0}+\mathcal{O}(d)$, where $\eta_{0}$ is $\eta_{c}$ with $u_{c}$ replaced by $u_{0}$. Similarly, $f_{0}$ is defined to be $f(u)$ with $u=u_{0}$. On the other hand, for the limit of large $d$ in (3.34), the pressure from (3.43) becomes [12]

$$
P \simeq \frac{2}{35}\left(\frac{\Gamma(1 / 5) \Gamma(3 / 10)}{\Gamma(1 / 2)}\right) d^{7 / 5} .
$$

Numerically, the relations between the pressure and the density of the multiquark matter for different values of $n_{s}$ are plotted in Figure 6 . This is consistent with the results of analytic calculations that $P \sim d^{2}$ for small $d$ and $P \sim d^{7 / 5}$ for large $d$. Since the relations are not sensitive to the change of $T$, therefore we present only the plots at $T=0.03$. The transition from small to large $d$ is apparent at $d_{c} \simeq 0.072$. We can also see the dependence of pressure on $n_{s}$ from the plots. The pressure of the multiquarks with larger $n_{s}$ is smaller for small $d$. On the other hand, the pressure of the multiquarks with smaller $n_{s}$ is merely slightly larger in the large $d$ limit. Actually, the pressure is nearly insensitive to the changing of $n_{s}$ for $d>d_{c}$ as is implied from (3.46).

From the differential of the free energy, the entropy density can be written as

$$
s=-\frac{\partial \mathscr{F}_{E}}{\partial T}
$$

where $\mathcal{F}_{E}$ is the free energy density which relates to the grand potential density as $\mathcal{F}_{E}=$ $\Omega+\mu d$. Using (3.41), the entropy density becomes

$$
s=\frac{\partial P}{\partial T}-\left(\frac{\partial \mu}{\partial T}\right) d
$$

Since the pressure $P$ and the contribution of D8-branes to the baryon chemical potential $(\mu-$ $\left.\mu_{\text {source}}\right)$ are insensitive to the changing of the temperature, the entropy density is dominated by the derivative of $\mu_{\text {source }}$ with respect to $T$, That is,

$$
s \simeq-\left(\frac{\partial \mu_{\text {source }}}{\partial T}\right) d \text {. }
$$




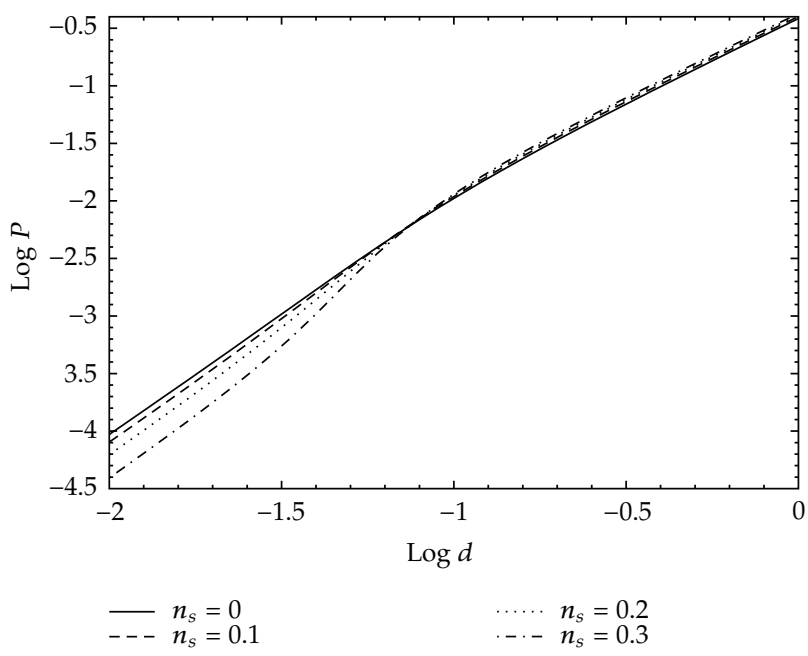

Figure 6: Pressure versus density of the multiquark phase in logarithmic scale at $T=0.03$, zoomed in around the transition region.

It is found numerically in [52] that $u_{c}$ is approximately constant with respect to the temperature range between the gluon deconfinement and the chiral symmetry restoration, we thus obtain

$$
\begin{aligned}
\frac{\partial \mu_{\text {source }}}{\partial T} & =\frac{\partial}{\partial T}\left(\frac{1}{3} u_{c} \sqrt{f\left(u_{c}\right)}+n_{s}\left(u_{c}-u_{T}\right)\right) \\
& \approx-\frac{\left(16 \pi^{2} / 9\right)^{3} T^{5}}{u_{0}^{2} \sqrt{1-\left(u_{T} / u_{0}\right)^{3}}}-n_{s} \frac{32 \pi^{2} T}{9},
\end{aligned}
$$

such that

$$
s \approx \frac{\left(16 \pi^{2} / 9\right)^{3} T^{5} d}{u_{0}^{2} \sqrt{1-\left(u_{T} / u_{0}\right)^{3}}}+n_{s} \frac{32 \pi^{2} T d}{9} .
$$

The entropy density has the temperature dependence $\sim T^{5}$ for small $n_{s}$, whereas it is dominated by the colour term, that is, $s \propto n_{s} T$, for larger $n_{s}$. This agrees with the numerical results shown in Figure 7. We already know that the free quarks and gluons in the XS-QGP have the entropy density scales as $T^{6}$ [48]. Intriguingly, the presence of the colour charges of multiquarks implies that the multiquark matter in the sQGP behave less like free particles with the weaker temperature dependence $s \sim n_{s} T$. Also confirmed numerically in Figure 7 is the linear dependence of the entropy density to the density $d$.

From (3.43), it is important to note that the pressure is mainly contributed from the flavour D8-brane part since $\mu_{\text {source }}$ is mostly constant with respect to the density. This is because the constant part of $\mu$ with respect to the density will cancel out when substituted into (3.43). Conversely, the entropy density is mainly contributed from the source term, namely, the vertex and radial strings.

Lastly, the dependence of the baryon chemical potential $\mu$ on $d$ is plotted in Figure 8 . The $\mu-d$ relation is found to be mostly independent of the temperature. It is found that the 


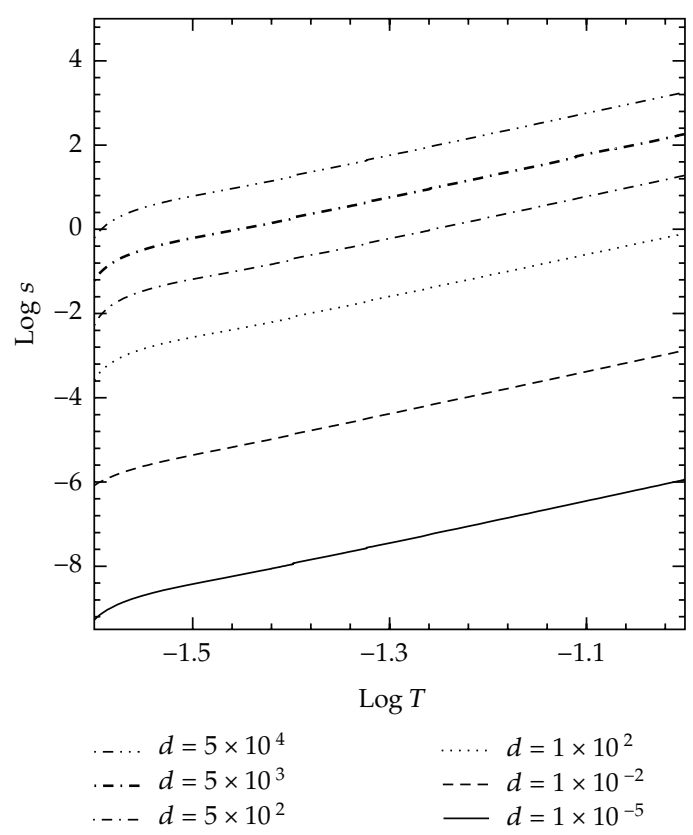

(a)

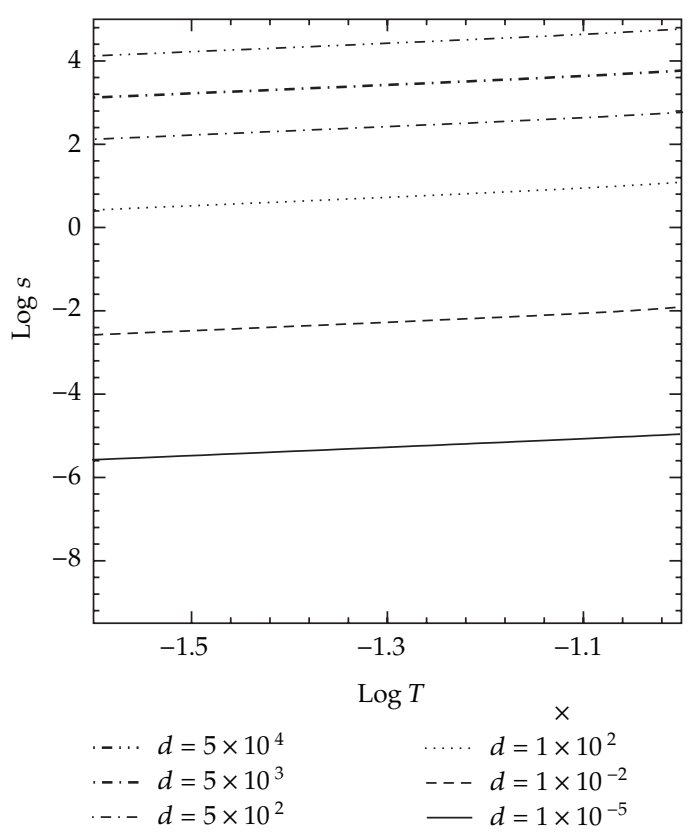

(b)

Figure 7: Entropy versus temperature of the multiquark phase in logarithmic scale for $n_{s}=0$ (a), 0.3 (b).

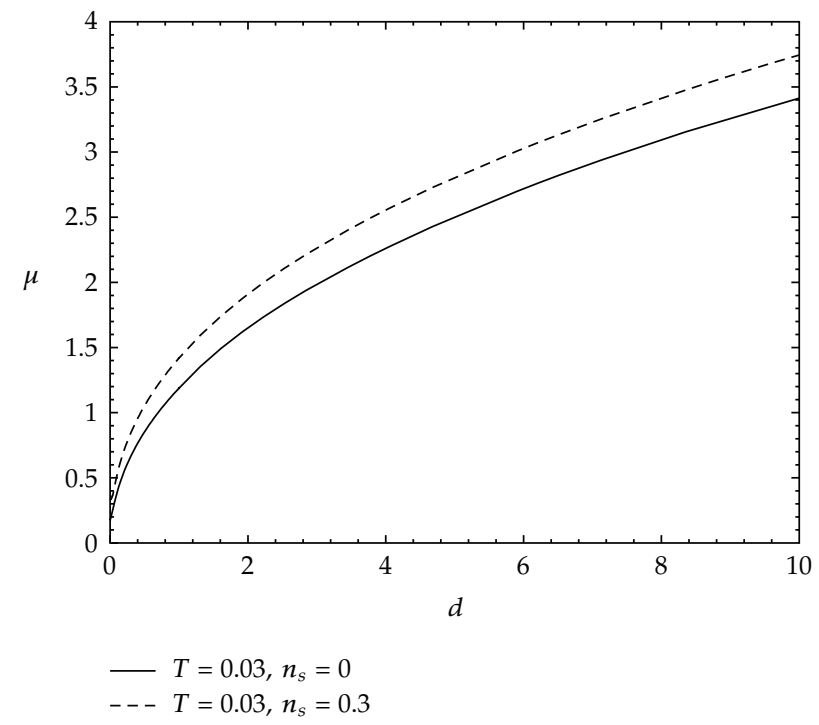

Figure 8: The baryon chemical potential versus number density of the multiquark phase at $T=0.03$.

relation can be well approximated by the power law with $\mu \sim d$ for small density and $\mu \sim d^{2 / 5}$ for large density. The difference indicates that the behaviour of multiquark quasiparticles is more like fermions as a consequence of the DBI action [48]. 


\section{Magnetic Properties of Holographic Multiquarks in the Quark-Gluon Plasma}

In the deconfined SS model, the multiquark phase has been shown to be the most thermodynamically preferred in the extremely dense and warm condition (in the temperature range above the deconfinement but below the chiral symmetry restoration). It is possible that the real dense and warm QCD soup also energetically prefers the multiquark phase in such condition. In the early universe, during the electroweak phase transition, the Higgs mechanism could create enormous magnetic fields in the boundary region between two domains with different vacuum expectation values [53]. These gigantic fields could have crucial impact on the phase transitions of the warm nuclear soup at later epoch. Collision of energetic charged particles at the hadron and heavy ion colliders could produce extremely large magnetic fields in the vicinity of the collision point. At RHIC and LHC, it has been estimated that the induced local magnetic fields could be as large as $10^{14-15}$ Tesla [54]. Finally, magnetic fields of order of $10^{10}$ Tesla could be produced by the magnetars on the large astrophysical scale [55]. Therefore, it is interesting to investigate the effects of extremely strong magnetic fields to the multiquark phase above the deconfinement temperature.

To mimic behaviour of the strongly coupled nuclear matter in the presence of a uniform magnetic field, we turn on another component of the $U(1) \subset U\left(N_{f}\right)$ field denoted as $a_{3}^{V}$. The nonnormalizable modes of $a_{3}^{V}$ are identified with the vector potential of the magnetic field $B$ (not to be confused with $B$ used in Section 2), defined in units of $1 / 2 \pi \alpha^{\prime}$. We choose the direction of the magnetic field so that

$$
a_{3}^{V}=B x_{2}
$$

As before, the baryon chemical potential $\mu$ of the corresponding gauge matter at the boundary is identified with the nonnormalizable mode of the DBI-gauge field by

$$
\mu=a_{0}^{V}(u \longrightarrow \infty)
$$

Additional sources of the baryonic charge in terms of the instanton and strings contribute the following action

$$
\begin{aligned}
S_{\text {source }} & =\mathcal{N} d\left(u_{c}\right)\left[\frac{1}{3} u_{c} \sqrt{f\left(u_{c}\right)}+n_{s}\left(u_{c}-\mathrm{u}_{T}\right)\right] \\
& \simeq \mathcal{N} \mu_{\text {source }}
\end{aligned}
$$

where $n_{s}=k_{r} / N_{c}$ is the number of radial strings in the unit of $1 / N_{c}$ as in the zero magnetic field case. The electric displacement, $d\left(u_{c}\right) \equiv \partial L /\left.\partial a_{0}^{V^{\prime}}\right|_{u_{c}}$, representing the baryonic charge density from the $\mathrm{D} 4$ at $u_{c}$ has been approximated to be $d$ (the exact value is $d-(3 / 2) B a_{1}^{A}(\infty)$ ). This action does not contain the gauge fields of the flavour branes and thus does not affect the equations of motion. However, it contributes tidal weight to the total configuration, pulling down the flavour branes closer to the horizon. The scale-fixing condition $L_{0}=1$ is determined by the equilibrium between this tidal weight of the additional sources (including the tension of the colour strings) and the tension of the flavour branes. The position $u_{c}$ of the tip of the connecting branes determined from this condition will also depend on the magnetic field in presence. 
The response of the flavour branes to the magnetic field is encoded in the axial $a_{1}^{A}$ component of the $U(1)$ induced through the Chern-Simons action of the branes in the bulk. In the boundary gauge theory, this corresponds to the axial anomaly described by the WessZumino-Witten action [56]. The non-normalizable mode of $a_{1}^{A}$ at the boundary is identified with the response of the chiral condensate to the external magnetic field, $a_{1}^{A}(u \rightarrow \infty) \equiv \nabla \varphi$, which we will call the pion gradient.

The D-brane and the Chern-Simons action of the configuration can be calculated straightforwardly to be

$$
\begin{gathered}
S_{\mathrm{D} 8}=\mathcal{N} \int_{u_{c}}^{\infty} d u u^{5 / 2} \sqrt{1+\frac{B^{2}}{u^{3}}} \sqrt{1+f(u)\left(a_{1}^{\prime A}\right)^{2}-\left(a_{0}^{\prime V}\right)^{2}+f(u) u^{3} x_{4}^{\prime 2}}, \\
S_{\mathrm{CS}}=-\frac{3}{2} \mathcal{N} \int_{u_{c}}^{\infty} d u\left(\partial_{2} a_{3}^{V} a_{0}^{V} a_{1}^{A^{\prime}}-\partial_{2} a_{3}^{V} a_{0}^{V^{\prime}} a_{1}^{A}\right),
\end{gathered}
$$

where $\mathcal{N}=N_{c} R_{\mathrm{D} 4}^{2} /\left(6 \pi^{2}\left(2 \pi \alpha^{\prime}\right)^{3}\right)$ defines the brane tension. To preserve the gauge invariance of the total action in the situation where the gauge transformation does not vanish at the spatial infinity, addition of surface terms effectively results in the factor $3 / 2$ in the ChernSimons action [57].

Appearance of horizon in the background spacetime connects classical behaviour of the bulk physics to the physics of the quantum gauge matter at the boundary in a holographic manner. The brane-bulk interaction provides a solid correspondence between bulk fields and operators of the gauge theory on the boundary. Classical solutions of the gauge fields on the D8- $\overline{\mathrm{D} 8}$ probe as well as its geometric configuration will describe physics of the dense strongly coupled nuclear matter in the presence of the external magnetic field in a holographic manner.

By conventional variational method, the equations of motion with respect to the gauge field components are given by the Euler-Lagrange equation with respect to the gauge field component $a_{0}^{V}, a_{1}^{A}$,

$$
\begin{aligned}
& \frac{\sqrt{u^{5}+B^{2} u^{2}} f(u) a_{1}^{\prime A}}{\sqrt{1+f(u)\left(a_{1}^{\prime A}\right)^{2}-\left(a_{0}^{\prime V}\right)^{2}+f(u) u^{3} x_{4}^{\prime 2}}}=j_{A}-\frac{3}{2} B \mu+3 B a_{0}^{V}, \\
& \frac{\sqrt{u^{5}+B^{2} u^{2}} a_{0}^{\prime V}}{\sqrt{1+f(u)\left(a_{1}^{\prime A}\right)^{2}-\left(a_{0}^{\prime V}\right)^{2}+f(u) u^{3} x_{4}^{\prime 2}}}=d-\frac{3}{2} B a_{1}^{A}(\infty)+3 B a_{1}^{A} .
\end{aligned}
$$

Note that $d, j_{A}$ are the baryon charge density and current density of the dual gauge matter at the boundary $(u \rightarrow \infty)$ given by

$$
\begin{aligned}
j^{\mu}(x, u \longrightarrow \infty) & \left.\equiv \frac{\delta S_{\mathrm{eom}}}{\delta A_{\mu}}\right|_{u \rightarrow \infty} \\
& \equiv\left(d, \vec{j}_{A}\right) .
\end{aligned}
$$


They can be presented with the gauge fields as

$$
\begin{aligned}
& d=\left.\frac{\sqrt{u^{5}+B^{2} u^{2}} a_{0}^{\prime V}}{\sqrt{1+f(u)\left(a_{1}^{\prime A}\right)^{2}-\left(a_{0}^{\prime V}\right)^{2}+f(u) u^{3} x_{4}^{\prime 2}}}\right|_{\infty}-\frac{3}{2} B a_{1}^{A}(\infty), \\
& j_{A}=\left.\frac{\sqrt{u^{5}+B^{2} u^{2}} f(u) a_{1}^{\prime A}}{\sqrt{1+f(u)\left(a_{1}^{\prime A}\right)^{2}-\left(a_{0}^{\prime V}\right)^{2}+f(u) u^{3} x_{4}^{\prime 2}}}\right|_{\infty}-\frac{3}{2} B \mu .
\end{aligned}
$$

The action does not explicitly depend on $x_{4}$, consequently the associate constant of motion allows us to rewrite $x_{4}^{\prime}(u)$ as the following:

$$
\left(x_{4}^{\prime}(u)\right)^{2}=\frac{1}{u^{3} f(u)}\left[\frac{u^{3}\left[f(u)\left(C(u)+D(u)^{2}\right)-\left(j_{A}-(3 / 2) B \mu+3 B a_{0}^{V}\right)^{2}\right]}{F^{2}}-1\right]^{-1},
$$

where

$$
F=\frac{u_{c}^{3} \sqrt{f\left(u_{c}\right)} \sqrt{f\left(u_{c}\right)\left(C\left(u_{c}\right)+D\left(u_{c}\right)^{2}\right)-\left(j_{A}-(3 / 2) B \mu+3 B a_{0}^{V}\left(u_{c}\right)\right)^{2}} x_{4}^{\prime}\left(u_{c}\right)}{\sqrt{1+f\left(u_{c}\right) u_{c}^{3} x_{4}^{\prime 2}\left(u_{c}\right)}},
$$

and $C(u) \equiv u^{5}+B^{2} u^{2}, D(u) \equiv d+3 B a_{1}^{A}(u)-3 B \nabla \varphi / 2$.

From the scale-fixing condition

$$
L_{0}=2 \int_{u_{c}}^{\infty} x_{4}^{\prime}(u) d u=1
$$

the position of the tip $u_{c}$ of the brane configuration is determined by the equilibrium of forces to be, (see the appendix),

$$
\left(x_{4}^{\prime}\left(u_{c}\right)\right)^{2}=\frac{1}{f_{c} u_{c}^{3}}\left[\frac{9}{d^{2}} \frac{\left(f_{c}\left(C_{c}+D_{c}^{2}\right)-\left(j_{A}-(3 / 2) B \mu+3 B a_{0}^{V}\left(u_{c}\right)\right)^{2}\right)}{\left(1+(1 / 2)\left(u_{T} / u_{c}\right)^{3}+3 n_{s} \sqrt{f_{c}}\right)^{2}}-1\right] .
$$

The introduction of the Chern-Simons interaction of the gauge fields to the magnetic field results in the dependence of $x_{4}^{\prime}$ on the gauge field $a_{0}^{V}, a_{1}^{A}$. It is consequently required to solve (4.6), (4.7), and (4.12) simultaneously. Since the physical parameters, $\mu, \nabla \varphi$, also depend on the gauge field components by $a_{0}^{V}(\infty)=\mu, a_{1}^{A}(\infty)=\nabla \varphi$, we need a triple-shooting algorithm to solve for the solutions numerically.

Under the boundary conditions $a_{0}^{V}\left(u_{c}\right)=\mu_{\text {source, }} a_{1}^{A}\left(u_{c}\right)=0$, the values of $u_{c}, \mu, \nabla \varphi$ are chosen so that they solve the equations of motion and satisfy $a_{0}^{V}(\infty)=\mu, a_{1}^{A}(\infty)=\nabla \varphi$. If the solutions also satisfy the scale fixing condition (4.12), we keep the solutions, otherwise we adjust the value of $u_{c}$ and repeat the shooting procedure. 


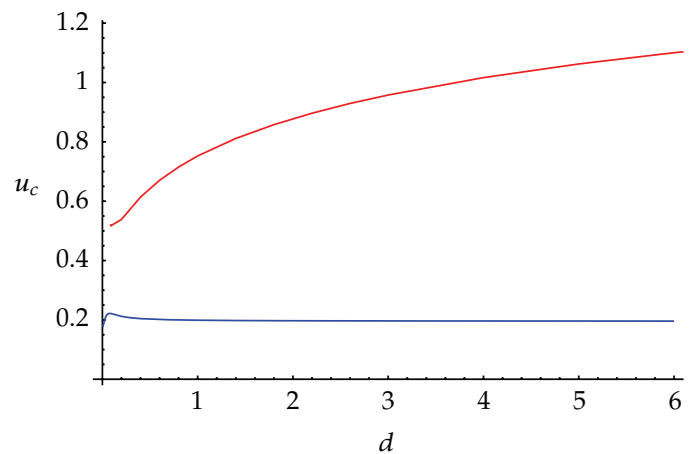

(a)

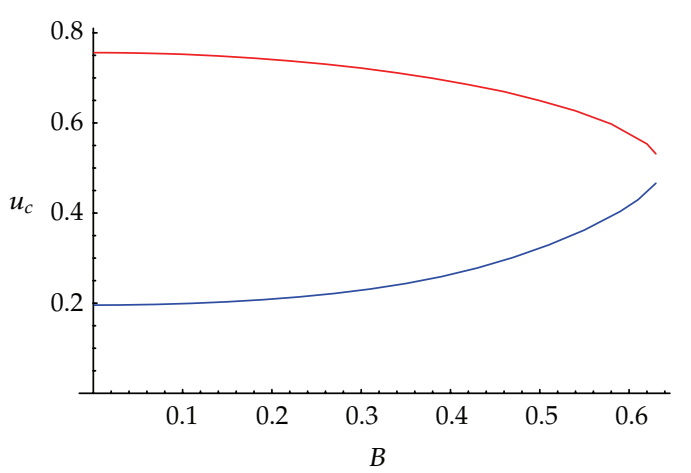

(b)

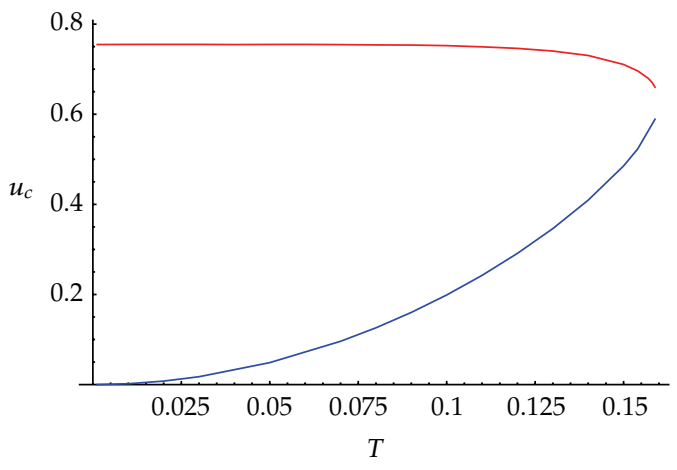

(c)

Figure 9: Position $u_{c}$ of the vertex for $n_{s}=0$ (normal baryon) and fixed $j_{A}=0$ as a function of (a) $d$ with fixed $B=0.10, T=0.10$, (b) $B$ with fixed $d=1, T=0.10$, (c) $T$ with fixed $B=0.10, d=1$. The lower (blue) line is the configuration A with $u_{c}$ close to $u_{T}$, and the upper (red) line is the configuration $\mathbf{B}$ with large separation between $u_{c}$ and $u_{T}$.

\subsection{Two Multiquark Configurations and the Multiquark Merging}

The numerical solutions obtained by the shooting algorithm reveal two possible multiquark configurations, one with small and one with large $u_{c}$. The small- $u_{c}$ configuration (configuration A) has longer stretch in the $u$-direction, therefore it contains higher gluon content and larger energy. The free energy of this configuration is consequently larger than the large- $u_{c}$ configuration (configuration $\mathbf{B}$ ) and becomes less energetically favoured. The relationships between $u_{c}$ and the baryonic density, the magnetic field, and the temperature are shown in Figure 9 (from [58]).

From Figure 9, the density dependence of the two configurations shows that as density increases, configurations $\mathbf{A}$ and $\mathbf{B}$ diverge from each other. They become two distinctive phases at large densities. On the other hand, the increase of magnetic field and temperature merges the two configurations together. At the critical field and/or temperature, configurations A and B merge and disappears (i.e., they do not satisfy the scale-fixing condition anymore).

In Figure 10, the chemical potential and the pion gradient response of the multiquark phase are plotted as functions of the magnetic field for the multiquark phase with the number of colour strings $n_{s}=0,0.1,0.2$. Magnetic merging occurs at higher field for the multiquarks with smaller $n_{s}$. Interestingly, the less-preferred configuration $\mathbf{A}$ has a negative 


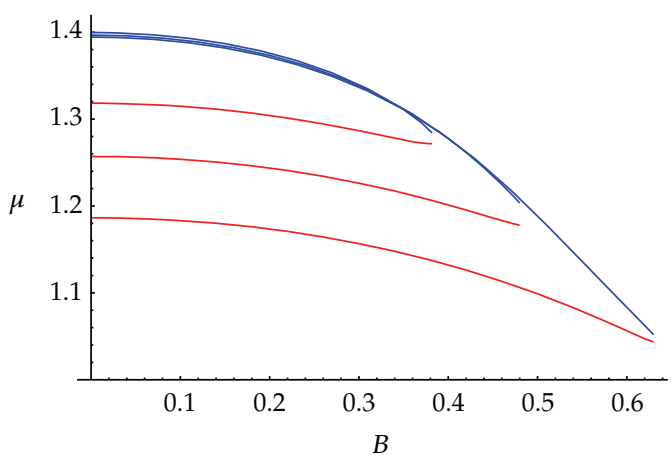

(a)

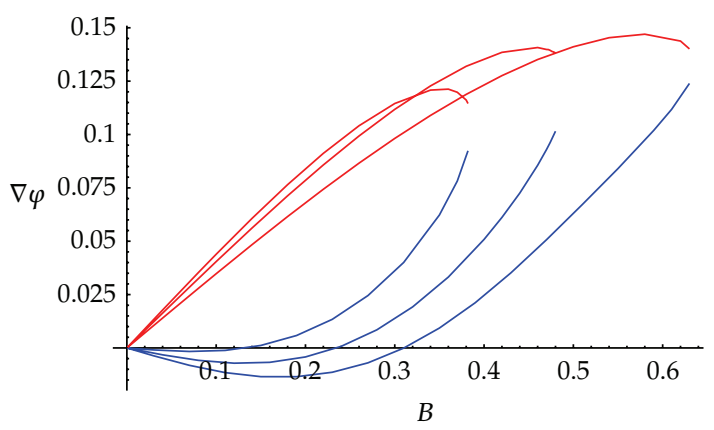

(b)

Figure 10: Comparison between the baryon chemical potential (a) and the pion gradient (b) as a function of $B$ at fixed $j_{A}=0, d=1, T=0.10$; for $n_{s}=0$ (normal baryon), the bottom graph; $n_{s}=0.10$, the middle graph; $n_{s}=0.20$, the top graph. The blue lines are the configuration $\mathbf{A}$ with $u_{c}$ close to $u_{T}$, and the red lines are the configuration $\mathbf{B}$ with large separation between $u_{c}$ and $u_{T}$.

linear response $\nabla \varphi$ to the magnetic field for small fields. Thorough investigation in [58] reveals that both multiquark configuration A, B are more thermodynamically preferred than the magnetized vacuum, and each configuration is stable under density fluctuations since

$$
\frac{\partial^{2} \mathcal{F}_{\mathrm{E}}}{\partial d^{2}}=\frac{\partial \mu}{\partial d}>0
$$

where the free energy $\mathcal{F}_{\mathrm{E}}$ will be defined in the next subsection.

We would like to emphasize that there are actually two possible multiquark phases for the deconfined nuclear matter at finite density. Even though phase $\mathbf{B}$ is more energetically preferred, both multiquark phases could coexist in general situation. Large magnetic field or high temperature could merge the two multiquark configurations into one. Remarkably once they merge, the multiquark can no longer exist since it does not satisfy the scale fixing condition. They would either turn into a multiquark configuration with larger density or a chiral-symmetric QGP. We will discuss more on the thermodynamic properties and phase diagram of the multiquark phases in the subsequent sections.

\subsection{Thermodynamic Properties of the $M Q-\nabla \varphi$ Phase}

The holographic principle conjectures that the partition function of the string theory in the bulk is equal to the partition function of the gauge theory on the boundary. The free energy of the gauge matter at the boundary is equivalent to the string action in the bulk, namely, the DBI action up to a periodicity factor [59]. The D8-brane action from (4.4) can be calculated to be

$$
S_{\mathrm{D} 8}=\mathcal{N} \int_{u_{c}}^{\infty} d u C(u) \sqrt{\frac{f(u)\left(1+f(u) u^{3} x_{4}^{\prime 2}\right)}{f(u)\left(C(u)+D(u)^{2}\right)-\left(j_{A}-(3 / 2) B \mu+3 B a_{0}^{V}\right)^{2}}} .
$$




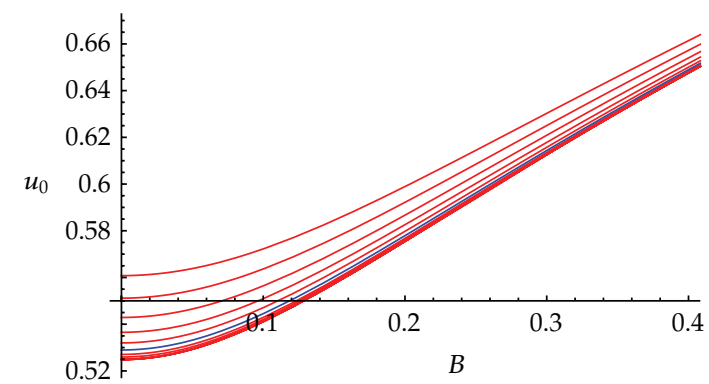

(a)

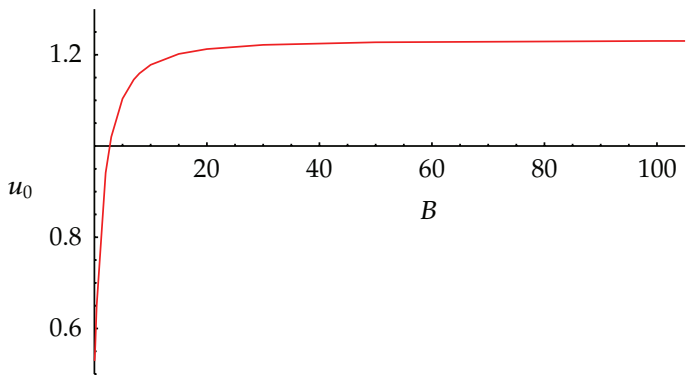

(b)

Figure 11: Relation between $u_{0}$ and external magnetic field $B$ of the vacuum for (a) the temperature $T=$ $0.02-0.15$, the upper lines have higher temperatures, (b) $u_{0}$ saturates to the approximate value of 1.23 at large field for all temperatures (only $T=0.10$ curve is shown here).

The action is divergent from the limit $u \rightarrow \infty$ and we need to regulate it using the action of the magnetized vacuum. For the magnetized vacuum, the field $a_{0}^{V}, a_{1}^{A}$, the baryon density, and chemical potential are set to zero giving

$$
S[\text { magnetized vacuum }]=\left.\int_{u_{0}}^{\infty} \sqrt{C(u)\left(1+f(u) u^{3} x_{4}^{\prime 2}\right)}\right|_{\text {vac }} d u,
$$

where

$$
\left.x_{4}^{\prime}(u)\right|_{\mathrm{vac}}=\frac{1}{\sqrt{f(u) u^{3}\left(f(u) u^{3} C(u) / f\left(u_{0}\right) u_{0}^{3} C\left(u_{0}\right)-1\right)}} .
$$

The position $u_{0}$ is the tip of the connecting brane configuration, since there is no source, $x_{4}^{\prime}\left(u_{0}\right) \rightarrow \infty$ and the branes and antibranes connect smoothly. Figure 11 shows $u_{0}$ as a function of the magnetic field and temperature. The value of $u_{0}$ converges to approximately 1.23 for high fields for all temperatures.

The regulated free energy at fixed density is then defined to be

$$
\mathcal{F}_{\mathrm{E}}(d, B) \equiv \Omega(\mu, B)+\mu d,
$$

where $\Omega(\mu, B)=S\left[a_{0}(u), a_{1}(u)\right]$ (e.o.m.) $-S[$ magnetized vacuum], and the total action $S\left[a_{0}(u), a_{1}(u)\right]\left(\right.$ e.o.m.) is given by $S_{\mathrm{D} 8}+S_{\mathrm{CS}}$.

The corresponding magnetization at fixed density is subsequently

$$
M(d, B)=-\left.\frac{\partial \mathcal{F}_{\mathrm{E}}(d, B)}{\partial B}\right|_{d}
$$

For $n_{s}=0,0.1,0.2$ we can plot the free energy and magnetization of the multiquark- $\nabla \varphi$ matter as a function of the magnetic field as shown in Figure 12. Configuration A has larger free energy and magnetization than configuration $\mathbf{B}$. The magnetic merging is clearly visible at critical fields. The critical field for multiquark with higher number of colour strings $n_{s}$ is smaller, reflecting less stability. The magnetization is approximately linear for small fields for 


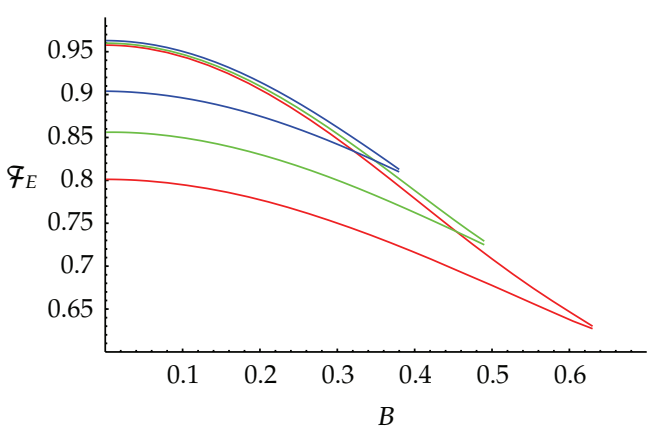

(a)

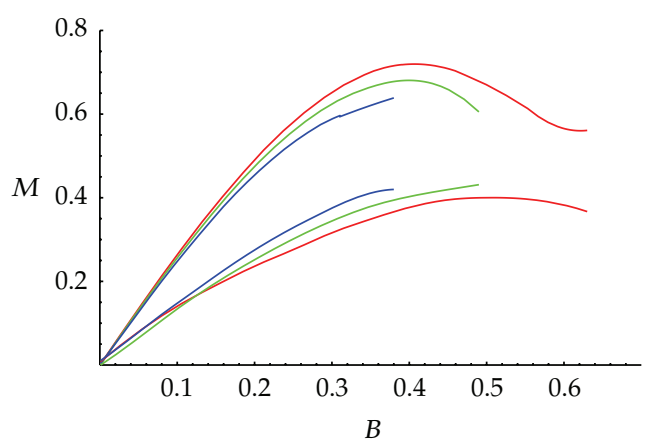

(b)

Figure 12: The free energy and magnetization of the multiquarks nuclear matter at fixed $j_{A}=0, d=1$, and $T=0.10$ for $n_{s}=0$ (red), 0.10 (green), 0.20 (blue). The upper lines are the configuration A with $u_{c}$ close to $u_{T}$, and the lower lines are the configuration $\mathbf{B}$ with large separation between $u_{c}$ and $u_{T}$.

both configurations. The free energy of configuration $\mathbf{A}$ is clearly larger than configuration $\mathbf{B}$, implying that it is less energetically preferred. Here and henceforth, we will focus our consideration to the multiquarks in configuration $\mathbf{B}$.

For moderate fields $B=0.05-0.15$, we can study the temperature dependence of the baryon chemical potential and the free energy of the MQ- $\nabla \varphi$ phase as shown in Figure 13. Remarkably, they inherit the temperature dependence from the factor $\sqrt{f(u)}=\sqrt{1-u_{T}^{3} / u^{3}}$ in the spacetime metric of the background SS model,

$$
\begin{aligned}
& \mu=\mu_{0}(d, B) \sqrt{1-\left(\frac{T}{T_{0}}\right)^{6}}, \\
& F=F_{0}(d, B) \sqrt{1-\left(\frac{T}{T_{0}}\right)^{6}},
\end{aligned}
$$

where for $d=1, B=0.10, \mu_{0}=1.1849, F_{0}=0.7976$, respectively. The best-fit values of $T_{0}$ for the chemical potential and the free energy are 0.269 and 0.233 , respectively. Note that the characteristic temperature $T_{0}$ of the chemical potential is slightly larger than the value of the free energy due to the additional temperature dependence of the $u_{c}$ in the free energy case [60]. It should be noted that the temperature dependence becomes significant for $T \gtrsim 0.10$.

When the magnetic field is applied to the multiquark phase, the chiral condensate responds to the field by developing the pion gradient $\nabla \varphi$ in the direction of the applied field. For moderate fields, the response is linear, $\nabla \varphi \propto B$, as we can see from Figure 10. The induced domain wall is stable among the multiquarks, carrying baryon density $d_{\nabla \varphi}=3 B \nabla \varphi / 2$ [56].

Figure 14 shows the relationship between the pion gradient and the magnetic field in the temperature range $T=0.02-0.15$. For $d=1$, the slope $m$ (or the linear response) of the response $\nabla \varphi$ to $B$ depends on the temperature approximately as $m=m_{0} \sqrt{1-\left(T / T_{0}\right)^{6}}$, and

$$
\nabla \varphi \simeq B m_{0} \sqrt{1-\left(\frac{T}{T_{0}}\right)^{6}}
$$

where $m_{0}=0.347, T_{0}=0.177$. 


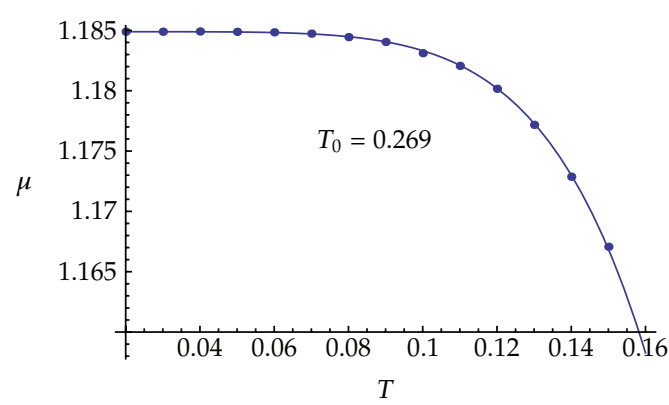

(a)

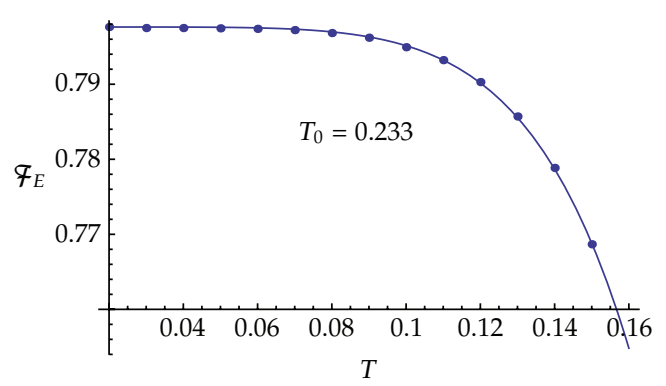

(b)

Figure 13: For $d=1, B=0.10$, (a) the baryon chemical potential as a function of $T$, the best-fit curve is in the form $\mu_{0} \sqrt{1-\left(T / T_{0}\right)^{6}}$ with $\mu_{0}=1.1849, T_{0}=0.269$; (b) the free energy as a function of $T$, the best-fit curve is in the form $F_{0} \sqrt{1-\left(T / T_{0}\right)^{6}}$ with $F_{0}=0.7976, T_{0}=0.233$. Other curves within the range $B=0.05-0.15$ can also be fitted well with the same $T_{0}$.

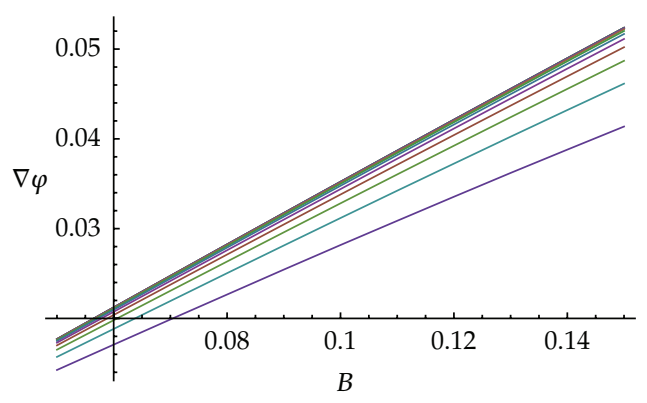

(a)

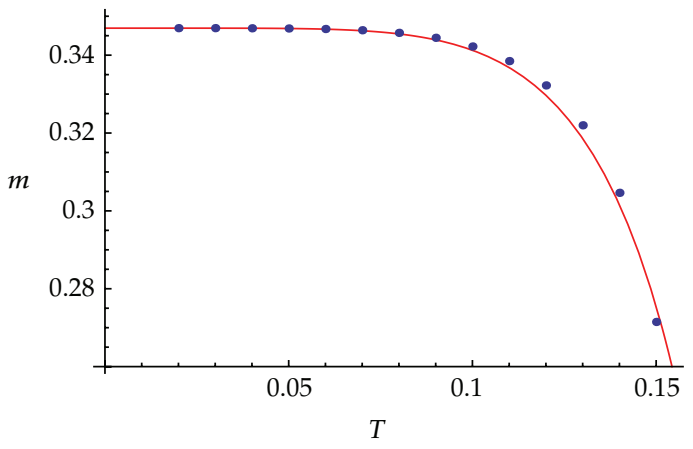

(b)

Figure 14: (a) The pion gradient versus magnetic field for $T=0.02-0.15$ at $d=1$, lower lines have higher temperatures. (b) The linear response or slope of the linear function between the pion gradient and the magnetic field as a function of the temperature for the range $B=0.05-0.15$ and density $d=1$. The red line is the best-fit curve in the form $m_{0} \sqrt{1-\left(T / T_{0}\right)^{6}}$ with $m_{0}=0.347, T_{0}=0.177$.

The pion gradient is induced naturally by the magnetic field as a result of axial anomaly in the boundary gauge theory. It can be described by the Wess-Zumino-Witten action in the chiral perturbation theory whilst the similar effect is represented by the ChernSimons action of the string theory in the bulk [56]. The pion gradient forms a domain wall which also carries baryonic charge and contributes to the total baryon density of the gauge matter. However, the population of the baryon density from the domain wall in the MQ- $\nabla \varphi$ phase decreases as the total density grows. This is shown in Figure 15. The ratio $R_{\nabla \varphi} \equiv d_{\nabla \varphi} / d=3 B \nabla \varphi$ can be approximated by a power law of the density as

$$
\begin{aligned}
R_{\nabla \varphi} & \simeq \text { (const.) } d^{-6 / 5}, \\
& \simeq \frac{3 B^{2} m_{0}}{2 d} \sqrt{1-\left(\frac{T}{T_{0}}\right)^{6}},
\end{aligned}
$$

as a result of (4.22). 


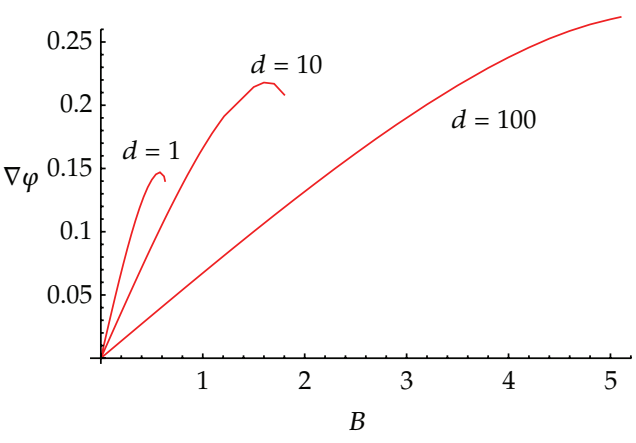

(a)

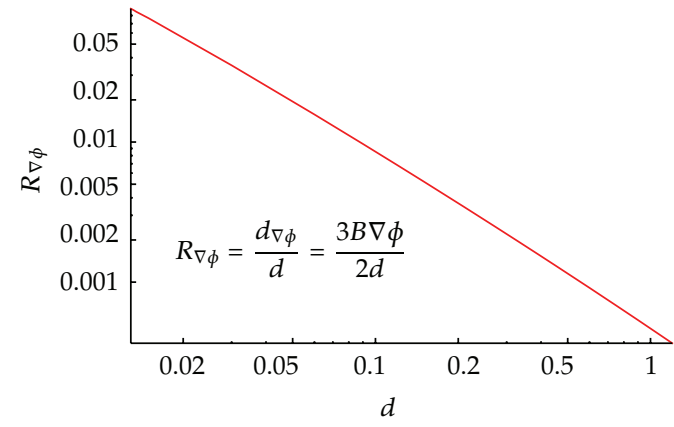

(b)

Figure 15: (a) The pion gradient as a function of $B$ for density $d=1,10,100$ at $T=0.10$. (b) The density ratio of the pion gradient with respect to the total baryon density of the multiquark phase at $B=0.10$ and $T=$ 0.10 in the double-log scale.

As the nuclear matter gets denser, the linear response of the chiral condensate to the magnetic field becomes smaller. The multiquark contribution to the baryonic charge density becomes dominant. In the extremely dense situation, the dominating phase of the deconfined nuclear matter is the multiquark with tiny mixture of the pion gradient when the magnetic field is present.

The remaining important issue is whether the MQ- $\nabla \varphi$ phase is more thermodynamically preferred than other phases such as the pure pion gradient and the chiral-symmetric QGP. Under which circumstances that the MQ- $\nabla \varphi$ phase is the most preferred and what the phase diagram of the deconfined nuclear matter in the SS model looks like are to be discussed.

\section{Comparison to Other Phases}

In the presence of the magnetic field, there are 4 possible nuclear phases in the deconfined SS model. For zero baryonic charge density and currents, there is a brane configuration corresponding to a magnetized vacuum. For nonzero baryon density, there are 3 possible brane configurations corresponding to 3 different nuclear phases:

$M Q-\nabla \varphi$ Phase

$j_{A}=0, \mu_{\text {source }}=a_{0}^{V}\left(u_{c}\right), \nabla \varphi=a_{1}^{A}(\infty), a_{1}^{A}\left(u_{c}\right)=0$,

\section{Pure Pion Gradient Phase}

The same conditions with the $\mathrm{MQ}-\nabla \varphi$ phase except, $\mu_{\text {source }}=0, a_{0}^{V}\left(u_{c}\right) \neq 0, d=$ $(3 / 2) B \nabla \varphi, x_{4}^{\prime}\left(u_{c}\right) \rightarrow \infty$,

\section{$x^{S-Q G P}$}

$x_{4}^{\prime}(u)=0$ and $\nabla \varphi=a_{1}^{A}(\infty)=0, \mu_{\text {source }}=a_{0}^{V}\left(u_{c}=u_{T}\right)=0, j_{A}=(3 / 2) B \mu$ (in order to satisfy the equation of motion at $u_{T}$ with $f\left(u_{T}\right)=0$ ).

The pure pion gradient phase corresponds to the brane configuration with no instanton at the tip $u_{c}$, where D8 and $\overline{\mathrm{D} 8}$ connect and thus $\mu_{\text {source }}=0$. The chiral condensate 


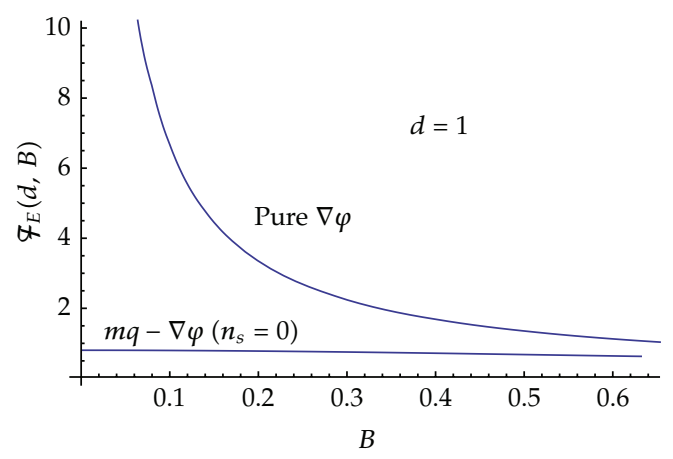

Figure 16: The free energy as a function of the density of the pure pion gradient phase compared to the multiquark $-\nabla \varphi$ phase at fixed $d=1.0, T=0$.

responds to the magnetic field by generating a gradient in the direction of the field. The induced domain wall carries baryonic charge density according to $d=3 B \nabla \varphi / 2$ [56]. Since there is no instanton at the tip, the branes connect smoothly just like in the case of vacuum and $x_{4}^{\prime}\left(u_{c}\right) \rightarrow \infty$.

The $x \mathrm{~S}$-QGP phase corresponds to the brane configuration with the D8 parallel to the $\overline{\mathrm{D}}$. Both branes never connect, and the distance between them in the direction $x_{4}$ is fixed to $L_{0}$. There is also no instanton source at the tip and $\mu_{\text {source }}=0$. Chiral symmetry demands that $a_{1}^{A}(\infty)=\nabla \varphi=0$. Remarkably, the equation of motion in the bulk automatically governs that the axial current is generated with $j_{A}=3 B \mu / 2$, a linear response of the magnetic field [57].

In the following subsections, we will compare the MQ- $\nabla \varphi$ with the pure pion gradient phase and subsequently the $x \mathrm{~S}-\mathrm{QGP}$ phase. By using the free energy at fixed density, it will be demonstrated that the pure pion gradient is always less energetically preferred than the MQ$\nabla \varphi$ for sufficiently large chemical potential. The second order phase transition lines between the 2 phases are drawn. The MQ- $\nabla \varphi$ phase is shown to be more preferred than the $\chi S$-QGP for moderate fields and temperatures. For very large field and temperature, the $x \mathrm{~S}$-QGP phase is the most thermodynamically preferred at a fixed density. Phase diagrams between the MQ$\nabla \varphi$ and the $x$ S-QGP for a fixed magnetic field and temperature are drawn and approximated with the power law.

\subsection{Multiquark-Domain Wall versus Pure $\nabla \varphi$ Phase}

The pure pion gradient phase has been explored in details in [56] using the effective field theory with the anomalous WZW action. The zero-temperature behaviour in the confined SS model and the bottom-up AdS/QCD model is studied in [47] and [61], respectively. Reference [62] compares the pure pion gradient phase with the $x \mathrm{~S}$-QGP by approximating $f(u) \simeq 1$ for the pure pion gradient phase. In this subsection, we will present the results from [63] where the full temperature dependence is taken into consideration.

Figure 16 shows the free energy at fixed density $d=1$ of the pure $\nabla \varphi$ and the MQ$\nabla \varphi$ phases as functions of the magnetic field. Apparently, the MQ- $\nabla \varphi$ is more energetically preferred than the pure pion gradient. At higher densities, since $\mu \sim d$ for the pure $\nabla \varphi$ [63] and $\mu \sim d^{n}, n<1$ for the MQ- $\nabla \varphi$, the dominant term $\mu d$ in the free energy will make the MQ$\nabla \varphi$ phase even more energetically preferred (with smaller free energy). It has been confirmed 


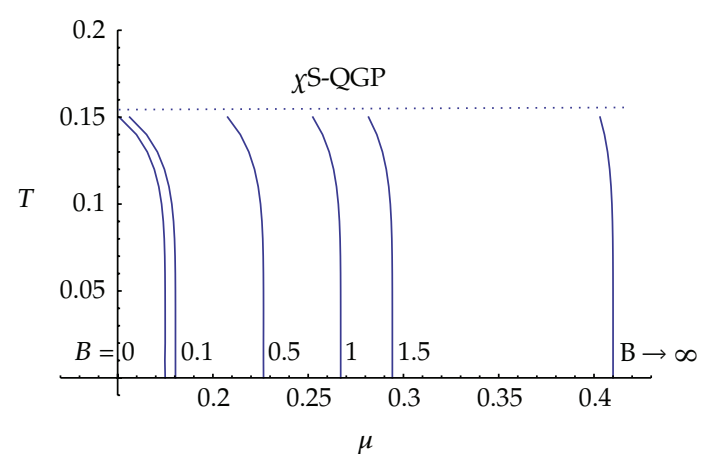

Figure 17: The onset chemical potential of the multiquark- $\nabla \varphi$ phase as a function of $T, B$ (for $B \rightarrow$ $\infty, u_{0}=1.23$ is used). These lines can be served as the transition lines between the $\nabla \varphi$ phase on the left and multiquark- $\nabla \varphi$ phase $\left(n_{s}=0\right)$ on the right. The dotted line represents schematic transition to the chiral-symmetric QGP phase.

numerically down to $d=0.1$ that the MQ- $\nabla \varphi$ is always more thermodynamically preferred than the pure $\nabla \varphi$ phase.

However, in the region of the parameter space, where the baryon chemical potential is smaller than the onset chemical potential of the multiquarks, only pure $\nabla \varphi$ phase can exist. The curve of the onset chemical potential of the multiquarks can thus be served as the second order transition line between the two phases. It depends on both the temperature and magnetic field in presence given by

$$
\mu_{\text {onset }}=\frac{1}{3} u_{c} \sqrt{f\left(u_{c}\right)}+n_{s}\left(u_{c}-u_{T}\right)
$$

where $u_{c}$ is a function of both $B$ and $T$. The phase diagram $(\mu, T)$ between the pure $\nabla \varphi$ and $\mathrm{MQ}-\nabla \varphi$ is shown in Figure 17.

The dotted line in the phase diagram represents a schematic transition from a chirally broken nuclear phases to the chiral-symmetric QGP phase. The transition from the pure $\nabla \varphi$ to the $x \mathrm{~S}$-QGP is investigated in [62]. Transition between the MQ- $\nabla \varphi$ and the $x \mathrm{~S}-\mathrm{QGP}$ will be discussed in the next subsection.

\subsection{Multiquark-Domain Wall versus $\chi^{S-Q G P}$ Phase}

In this subsection, we explore the phase diagram of the MQ- $\nabla \varphi$ and the $\chi \mathrm{S}-\mathrm{QGP}$ phases. For a fixed density, the baryon chemical potential and the free energy of each phase can be plotted as in Figure $18[58,60]$. The MQ- $\nabla \varphi$ is energetically preferred for small and intermediate fields for a fixed density. As the field increases further, the chiral-symmetric QGP becomes more favourable. At even larger fields, the curve of the $x \mathrm{~S}$-QGP has a break signifying a phase transition to the lowest Landau level $[62,64]$.

The phase diagrams $(d, B)$ for fixed temperature and $(d, T)$ for fixed magnetic field are presented in Figure 19. At given magnetic field and temperature, the MQ- $\nabla \varphi$ phase is more energetically preferred for a sufficiently large density. Dense nuclear matter prefers to form multiquark states even in the presence of the magnetic field. Nevertheless, for a given density, sufficiently high field and temperature will make the MQ- $\nabla \varphi$ phase less preferred than the $x$ S-QGP. 


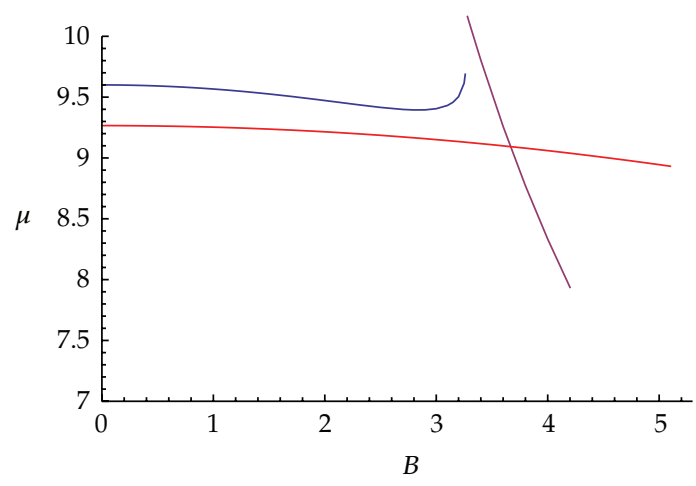

(a)

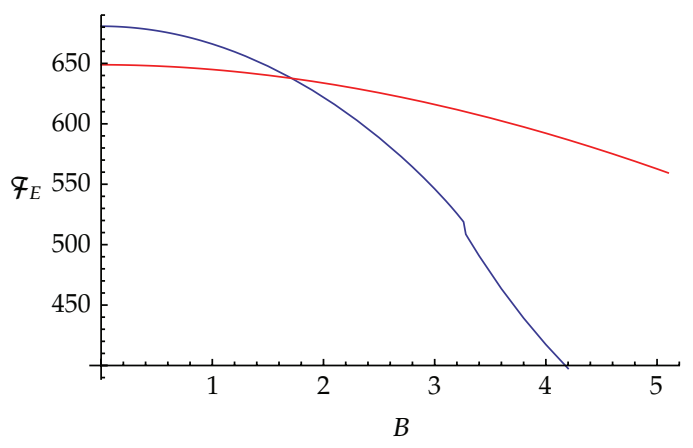

(b)

Figure 18: For the dense multiquark with $d=100, T=0.10$, (a) the chemical potential, (b) the free energy as a function of $B$. The multiquark curves in red are compared with the $x \mathrm{~S}$-QGP curves in blue for the chemical potential and the free energy.

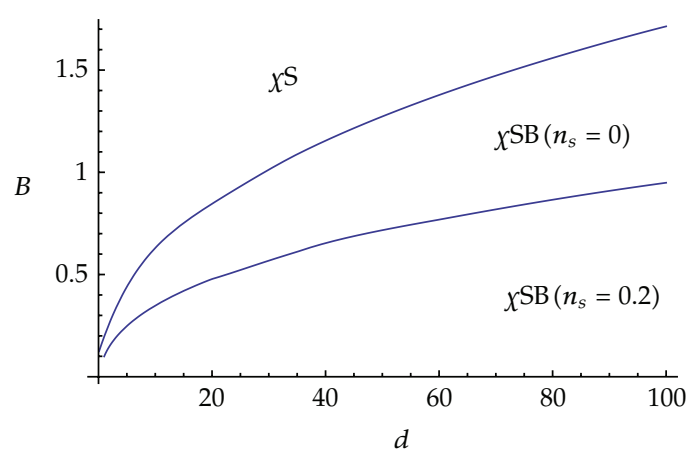

(a)

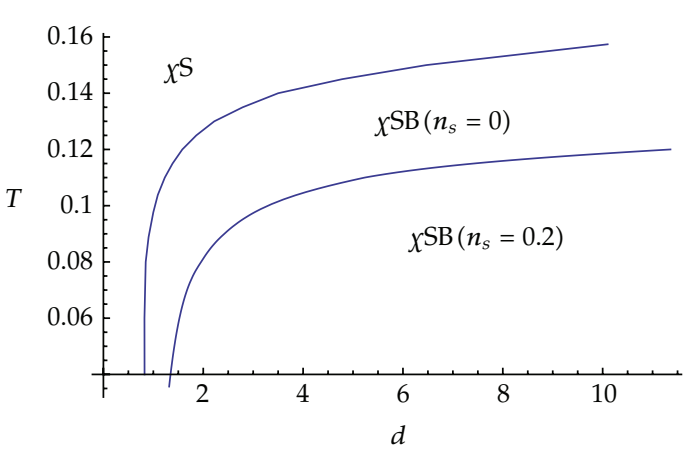

(b)

Figure 19: The phase diagram of the dense nuclear phases involving multiquarks when gluons are deconfined for (a) $T=0.10$ and (b) $B=0.20$. The chiral-symmetric quark-gluon plasma and the chirally broken $\mathrm{MQ}-\nabla \varphi$ phase are represented by $\chi \mathrm{S}$ and $\chi \mathrm{SB}$, respectively, $n_{S}$ is the number of colour strings in fractions of $1 / N_{c}$.

The transition lines between the $\chi \mathrm{S}$-QGP and the MQ- $\nabla \varphi$ phases in the $(d, B)$ phase diagram can be approximated with a power law,

$$
B \sim d^{0.438(0.436)}
$$

for multiquarks with $n_{S}=0(0.2)$ at $T=0.10$. This is weaker than the power-law $B \sim d^{2 / 3}$ of the $x \mathrm{~S}$-QGP transition to the lowest Landau level studied in [64]. On the other hand, the transition line in the $(d, T)$ phase diagram is weaker than a logarithm, yet still an increasing function of $d$. 


\section{Conclusions}

In this paper, we review the development of the holographic multiquark states in the deconfined quark-gluon plasma. We discuss their physical properties such as the binding energy, the screening length, the thermodynamical properties and the equation of state. Using the Sakai-Sugimoto model, we also explore the possible phase diagram of the multiquark matter. The multiquark phase is the most energetically preferred when the density and/or the baryon chemical potential is sufficiently large and the temperature is not too high. Even though the multiquark states with colour degrees of freedom are less preferred than the colour-singlet baryons, they are more energetically favoured than the other phases in such dense condition.

The magnetic properties and the magnetic phase diagram of the multiquark matter are subsequently reviewed. There are 2 possible holographic multiquark configurations, both of them are stable under density fluctuations. High magnetic field and temperature merge the 2 configurations into one. Once they merge, they transit to the multiquark configurations with larger densities, or to the chiral-symmetric QGP phase. In the region of the parameter space with sufficiently large densities and moderate fields at a fixed temperature, the magnetized multiquark phase is the most thermodynamically preferred. For a fixed magnetic field, sufficiently high temperature will melt the multiquarks into quarks and gluons regardless of the density.

In the region of parameter space with small density and baryon chemical potential ( $\mu<\mu_{\text {onset }}$ of the multiquarks), another magnetized nuclear phase called the pure pion gradient is dominant. When chiral symmetry is broken, an external strong magnetic field could induce a response of the chiral condensate in the direction of the applied field. The generated pion gradient also carries baryonic charge density and the corresponding chemical potential. However, once $\mu>\mu_{\text {onset }}$ of the multiquarks, the multiquark phase is always energetically preferred than the pure pion gradient. Inevitably, the pion gradient is also induced in the multiquark matter under the external field and render the multiquark matter in a mixed $\mathrm{MQ}-\nabla \varphi$ phase. The population of the pion gradient in the mixed phase is found to be a decreasing function with respect to the baryon density.

\section{Appendix}

\section{Force Condition of the Multiquark Configuration}

The forces on the D4-brane in the flavour D8-branes are balanced among three forces from the tidal weight of the D4-brane, the force from the strings attached to the D4, and the force from the D8-branes. Varying the total action with respect to $u_{c}$ gives the surface term. Together with the scale-fixing condition $2 \int_{u_{c}}^{\infty} d u x_{4}^{\prime}(u)=L_{0}=1$, we obtain [48]

$$
x_{4}^{\prime}\left(u_{c}\right)=\left.\frac{\left(\widetilde{L}\left(u_{c}\right)-\left(\partial S_{\text {source }} / \partial u_{c}\right)\right)}{\partial \widetilde{S} / \partial x_{4}^{\prime}}\right|_{u_{c}},
$$

as the condition on $u_{c}$. 
The Legendre transformed action is given by

$$
\begin{aligned}
\widetilde{S}= & \int_{u_{c}}^{\infty} \tilde{L}\left(x_{4}^{\prime}(u), d\right) d u \\
= & \mathcal{N} \int_{u_{c}}^{\infty} d u \sqrt{\frac{1}{f(u)}+u^{3} x_{4}^{\prime 2}} \\
& \times \sqrt{f(u)\left(C(u)+D(u)^{2}\right)-\left(j_{A}-\frac{3}{2} B \mu+3 B a_{0}^{V}\right)^{2}},
\end{aligned}
$$

where $C(u) \equiv u^{5}+B^{2} u^{2}, D(u) \equiv d+3 B a_{1}^{A}(u)-3 B \nabla \varphi / 2$. It is calculated by performing Legendre transformation with respect to $a_{0}^{V^{\prime}}$ and $a_{1}^{A \prime}$, respectively. Note that the ChernSimons action is also included in the total action during the transformations.

The Chern-Simons term with the derivatives $a^{V \prime}, a^{A \prime}$ eliminated is

$$
S_{\mathrm{CS}}=-\mathcal{N} \frac{3}{2} B \int_{u_{c}}^{\infty} d u \frac{\left(a_{0}^{V}\left(j_{A}-(3 / 2) B \mu+3 B a_{0}^{V}\right)-f(u) D(u) a_{1}^{A}\right) \sqrt{1 / f(u)+u^{3} x_{4}^{\prime 2}}}{\sqrt{f(u)\left(C(u)+D(u)^{2}\right)-\left(j_{A}-(3 / 2) B \mu+3 B a_{0}^{V}\right)^{2}}} .
$$

Lastly, in order to compute $x_{4}^{\prime}\left(u_{c}\right)$ we consider the source term [12]

$$
\begin{aligned}
S_{\text {source }} & =\mathcal{N} d\left(u_{c}\right)\left[\frac{1}{3} u_{c} \sqrt{f\left(u_{c}\right)}+n_{s}\left(u_{c}-u_{T}\right)\right] \\
& \simeq \mathcal{N} \mu_{\text {source }}
\end{aligned}
$$

where $n_{s}=k_{r} / N_{c}$ is the number of radial strings in the unit of $1 / N_{c}$. We have approximated the electric displacement at the position of the D4 brane source, $d\left(u_{c}\right) \equiv-\partial L /\left.\partial a_{0}^{V^{\prime}}\right|_{u_{c}}=d-$ $(3 / 2) B a_{1}^{A}(\infty)$ with $d$. to obtain

From (A.1), (A.2), (A.3), (A.5), and setting $a_{0}^{V}\left(u_{c}\right)=\mu_{\text {source, }} a_{1}^{A}\left(u_{c}\right)=0$ we can solve

$$
\left(x_{4}^{\prime}\left(u_{c}\right)\right)^{2}=\frac{1}{f_{c} u_{c}^{3}}\left[\frac{9}{d^{2}} \frac{\left(f_{c}\left(C_{c}+D_{c}^{2}\right)-\left(j_{A}-(3 / 2) B \mu+3 B a_{0}^{V}\left(u_{c}\right)\right)^{2}\right)}{\left(1+(1 / 2)\left(u_{T} / u_{c}\right)^{3}+3 n_{s} \sqrt{f_{c}}\right)^{2}}-1\right],
$$

where $f_{c} \equiv f\left(u_{c}\right), C_{c} \equiv C\left(u_{c}\right), D_{c} \equiv D\left(u_{c}\right)$.

When we fix the parameter $n_{s}$, the temperature $T$, the baryon density $d$, the axial current $j_{A}=0$ (by minimizing the action with respect to $a_{1}^{A}(\infty)$ ), and setting $a_{1}^{A}\left(u_{c}\right)=$ $0, a_{0}^{V}\left(u_{c}\right)=\mu_{\text {source, }}$ then the position $u_{c}$ of the D4-brane is completely determined as a function of the magnetic field $B$. Once the equations of motion are solved, the value of $\mu=a_{0}^{V}(\infty)$ and $a_{1}^{A}(\infty)$ are determined.

In the case of no magnetic field and finite baryon density, the force balance condition at the tip can be obtained simply by using (A.6). It can be done by setting all spatial components 
of bulk $U(1)$ gauge fields to be zero, leaving only the time component nonvanishing. This results in $C_{c}=u_{c}^{5}$ and $D_{c}=d$. Therefore, we obtain

$$
\left(x_{4}^{\prime}\left(u_{c}\right)\right)^{2}=\frac{1}{f_{c} u_{c}^{3}}\left[\frac{9}{d^{2}} \frac{f_{c}\left(u_{c}^{5}+d^{2}\right)}{\left(1+(1 / 2)\left(u_{T} / u_{c}\right)^{3}+3 n_{s} \sqrt{f_{c}}\right)^{2}}-1\right],
$$

implying the force balance condition at the tip.

\section{Acknowledgments}

Ekapong Hirunsirisawat is supported in part by the Commission on Higher Education (CHE), Thailand, under the Program Strategic Scholarships for Frontier Research Network for the Thai Doctoral Degree Program for this research. Piyabut Burikham is supported in part by the Toray Science Foundation, Japan (TSF), the Thailand Research Fund (TRF), and Commission on Higher Education (CHE) under Grant RMU5380048 and also by the Thailand Center of Excellence in Physics (ThEP). Piyabut Burikham and Ekapong Hirunsirisawat are also supported in part by Research Strategic (A1B1), Research Funds from the Faculty of Science, Chulalongkorn University.

\section{References}

[1] V. Khachatryan, A. M. Sirunyan, A. Tumasyan et al., "Observation of long-range, near-side angular correlations in proton-proton collisions at the LHC," Journal of High Energy Physics, vol. 2010, no. 9, pp. 1-38, 2010.

[2] E. Laermann and O. Philipsen, "Lattice QCD at finite temperature," Annual Review of Nuclear and Particle Science, vol. 53, pp. 163-198, 2003.

[3] T. Umeda, R. Katayama, O. Miyamura, and H. Matsufuru, "The study of charmonia near the deconfining transition on an anisotropic lattice with $\mathrm{O}(\mathrm{a})$ improved quark action," International Journal of Modern Physics A, vol. 16, no. 12, pp. 2215-2241, 2001.

[4] M. Asakawa and T. Hatsuda, "J/ $\psi$ and $\eta c$ in the deconfined plasma from lattice QCD," Physical Review Letters, vol. 92, no. 1, Article ID 012001, 4 pages, 2004.

[5] H. Iida, T. Doi, N. Ishii, and H. Suganuma, "J/Psi at high temperatures in anisotropic lattice QCD," POS, vol. LAT2005, article 184, 2006.

[6] J. M. Maldacena, "The Large N limit of superconformal field theories and supergravity," Advances in Theoretical and Mathematical Physics, vol. 2, pp. 231-252, 1998.

[7] E. Witten, "Anti-de Sitter space and holography," Advances in Theoretical and Mathematical Physics, vol. 2, pp. 253-291, 1998.

[8] E. Witten, "Anti-de Sitter space, thermal phase transition, and confinement in gauge theories," Advances in Theoretical and Mathematical Physics, vol. 2, no. 3, pp. 505-532, 1998.

[9] E. Witten, "Baryons and branes in anti de Sitter space," Journal of High Energy Physics, vol. 2, no. 7, 1998.

[10] D. J. Gross and H. Ooguri, "Aspects of large N gauge theory dynamics as seen by string theory," Physical Review D, vol. 58, no. 10, Article ID 106002, 12 pages, 1998.

[11] A. Brandhuber, N. Itzhaki, J. Sonnenschein, and S. Yankielowicz, “Baryons from supergravity,” Journal of High Energy Physics, vol. 2, no. 7, article 020, 1998.

[12] P. Burikham, A. Chatrabhuti, and E. Hirunsirisawat, "Exotic multi-quark states in the deconfined phase from gravity dual models," Journal of High Energy Physics, vol. 2009, no. 5, article 006, 2009.

[13] T. Sakai and S. Sugimoto, "Low energy hadron physics in holographic QCD," Progress of Theoretical Physics, vol. 113, no. 4, pp. 843-882, 2005.

[14] T. Sakai and S. Sugimoto, "More on a holographic dual of QCD," Progress of Theoretical Physics, vol. 114, no. 5, pp. 1083-1118, 2005. 
[15] R. L. Jaffe, “Multiquark hadrons. II. Methods," Physical Review D, vol. 15, no. 1, pp. 281-289, 1977.

[16] R. L. Jaffe, "Multiquark hadrons. I. Phenomenology of $Q^{2} \bar{Q}^{2}$ mesons," Physical Review D, vol. 15, no. 1, pp. 267-280, 1977.

[17] R. L. Jaffe, "Perhaps a stable dihyperon," Physical Review Letters, vol. 38, no. 5, pp. 195-198, 1977.

[18] R. L. Jaffe, "Exotica," Physics Reports, vol. 409, no. 1, pp. 1-45, 2005.

[19] J. Adams, M. M. Aggarwal, Z. Ahammed et al., "Experimental and theoretical challenges in the search for the quark-gluon plasma: the STAR Collaboration's critical assessment of the evidence from RHIC collisions," Nuclear Physics A, vol. 757, no. 1-2, pp. 102-183, 2005.

[20] B. B. Back, M. D. Baker, M. Ballintijn et al., "The PHOBOS perspective on discoveries at RHIC," Nuclear Physics A, vol. 757, no. 1-2, pp. 28-101, 2005.

[21] K. Adcox, S. S. Adler, S. Afanasiev et al., "Formation of dense partonic matter in relativistic nucleusnucleus collisions at RHIC: experimental evaluation by the PHENIX Collaboration," Nuclear Physics A, vol. 757, no. 1-2, pp. 184-283, 2005.

[22] I. Arsene, I. G. Bearden, D. Beavis et al., "Quark-gluon plasma and color glass condensate at RHIC? The perspective from the BRAHMS experiment," Nuclear Physics A, vol. 757, no. 1-2, pp. 1-27, 2005.

[23] J. Liao and E. V. Shuryak, "Polymer chains and baryons in a strongly coupled quark-gluon plasma," Nuclear Physics A, vol. 775, no. 3-4, pp. 224-234, 2006.

[24] E. V. Shuryak and I. Zahed, "Rethinking the properties of the quark-gluon plasma at Tc $<\mathrm{T}<4 \mathrm{Tc}_{\mathrm{c}}$, Physical Review C, vol. 70, no. 2, Article ID 21901, pp. 1-4, 2004.

[25] E. V. Shuryak and I. Zahed, "Toward a theory of binary bound states in the quark-gluon plasma," Physical Review D, vol. 70, no. 5, Article ID 054507, 16 pages, 2004.

[26] Y. Imamura, "Baryon mass and phase transitions in large N gauge theory," Progress of Theoretical Physics, vol. 100, no. 6, pp. 1263-1272, 1998.

[27] K. Ghoroku and M. Ishihara, "Baryons with D5-brane vertex and k-quark states," Physical Review D, vol. 77, no. 8, Article ID 086003, 11 pages, 2008.

[28] K. Ghoroku, M. Ishihara, A. Nakamura, and F. Toyoda, "Multiquark baryons and color screening at finite temperature," Physical Review D, vol. 79, no. 6, 11 pages, 2009.

[29] M. Bando, T. Kugo, A. Sugamoto, and S. Terunuma, "Pentaquark baryons in string theory," Progress of Theoretical Physics, vol. 112, no. 2, pp. 325-355, 2004.

[30] W. Y. Wen, "Multi-quark potential from AdS/QCD," International Journal of Modern Physics, vol. 23, pp. 4533-4543, 2008.

[31] M. V. Carlucci, F. Giannuzzi, G. Nardulli, M. Pellicoro, and S. Stramaglia, “AdS-QCD quark-antiquark potential, meson spectrum and tetraquarks," European Physical Journal C, vol. 57, no. 3, pp. 569-578, 2008.

[32] H. Forkel, "Light scalar tetraquarks from a holographic perspective," Physics Letters, Section B, vol. 694 , no. 3, pp. 252-257, 2010.

[33] K. Ghoroku, A. Nakamura, T. Taminato, and F. Toyoda, "Holographic penta and hepta quark state in confining gauge theories," Journal of High Energy Physics, vol. 2010, no. 8, article 007, 2010.

[34] J. Polchinski and M. J. Strassler, "The string dual of a confining four-dimensional gauge theory," http://arxiv.org/abs/hep-th/0003136.

[35] I. R. Klebanov and M. J. Strassler, "Supergravity and a confining gauge theory: duality cascades and xSB-resolution of naked singularities," Journal of High Energy Physics, vol. 4, no. 8, pp. 21-35, 2000.

[36] J. Maldacena and C. Nuñez, "Towards the large N limit of pure N = 1 super Yang-Mills theory," Physical Review Letters, vol. 86, no. 4, pp. 588-591, 2001.

[37] O. Aharony, J. Sonnenschein, and S. Yankielowicz, "A holographic model of deconfinement and chiral symmetry restoration," Annals of Physics, vol. 322, no. 6, pp. 1420-1443, 2007.

[38] K. Peeters and M. Zamaklar, "The string/gauge theory correspondence in QCD," European Physical Journal, vol. 152, no. 1, pp. 113-138, 2007.

[39] C. G. Callan, A. Güijosa, and K. G. Savvidy, "Baryons and string creation from the 5-brane worldvolume action," Nuclear Physics B, vol. 547, no. 1-2, pp. 127-142, 1999.

[40] C. G. Callan, A. Güijosa, K. G. Savvidy, and O. Tafjord, "Baryons and flux tubes in confining gauge theories from brane actions," Nuclear Physics B, vol. 555, no. 1-2, pp. 183-200, 1999.

[41] J. Maldacena, "Wilson loops in large N field theories," Physical Review Letters, vol. 80, no. 22, pp. 4859-4862, 1998.

[42] S. W. Hawking, "The path integral approach to quantum gravity," in General Relativity:An Einstein Centenary Survey, S. W. Hawking and W. Israel, Eds., pp. 746-789, Cambridge University Press, New York, NY, USA, 1979. 
[43] S. Kobayashi, D. Mateos, S. Matsuura, R. C. Myers, and R. M. Thomson, "Holographic phase transitions at finite baryon density," Journal of High Energy Physics, vol. 2007, no. 2, article 016, 2007.

[44] A. Chamblin, R. Emparan, C. V. Johnson, and R. C. Myers, "Charged AdS black holes and catastrophic holography," Physical Review D, vol. 60, no. 6, 17 pages, 1999.

[45] A. Chamblin, R. Emparan, C. V. Johnson, and R. C. Myers, "Holography, thermodynamics, and fluctuations of charged AdS black holes," Physical Review D, vol. 60, no. 10, pp. 1-20, 1999.

[46] D. Mateos, S. Matsuura, R. C. Myers, and R. M. Thomson, "Holographic phase transitions at finite chemical potential," Journal of High Energy Physics, vol. 2007, no. 11, article 085, 2007.

[47] O. Bergman, G. Lifschytz, and M. Lippert, "Response of holographic QCD to electric and magnetic fields," Journal of High Energy Physics, vol. 2008, no. 5, article 007, 2008.

[48] O. Bergman, M. Lippert, and G. Lifschytz, "Holographic nuclear physics," Journal of High Energy Physics, vol. 2007, no. 11, article 056, 2007.

[49] J. Polchinski, String Theory. Vol. 2: Superstring Theory and Beyond.

[50] M. R. Douglas, “Branes within branes," http://arxiv.org/abs/hep-th/9512077.

[51] N. Horigome and Y. Tanii, "Holographic chiral phase transition with chemical potential," Journal of High Energy Physics, vol. 2007, no. 1, article 072, 2007.

[52] P. Burikham, E. Hirunsirisawat, and S. Pinkanjanarod, "Thermodynamic properties of holographic multiquark and the multiquark star," Journal of High Energy Physics, vol. 2010, no. 6, article 040, 2010.

[53] T. Vachaspati, "Magnetic fields from cosmological phase transitions," Physics Letters, Section B, vol. 265, no. 3-4, pp. 258-261, 1991.

[54] D. E. Kharzeev, L. D. McLerran, and H. J. Warringa, "The effects of topological charge change in heavy ion collisions: 'Event by event P and CP violation'," Nuclear Physics A, vol. 803, no. 3-4, pp. 227-253, 2008.

[55] R. C. Duncan and C. Thompson, "Formation of very strongly magnetized neutron stars: implications for gamma-ray bursts," Astrophysical Journal, vol. 392, no. 1, pp. L9-L13, 1992.

[56] D. T. Son and M. A. Stephanov, "Axial anomaly and magnetism of nuclear and quark matter," Physical Review D, vol. 77, no. 1, Article ID 014021, 9 pages, 2008.

[57] O. Bergman, G. Lifschytz, and M. Lippert, "Magnetic properties of dense holographic QCD," Physical Review D, vol. 79, no. 10, 2009.

[58] P. Burikham, "Magnetic properties of holographic multiquarks in the quark-gluon plasma," Journal of High Energy Physics, vol. 2010, no. 4, article 045, 2010.

[59] O. Aharony, S. S. Gubser, J. Maldacena, H. Ooguri, and Y. Oz, "Large N field theories, string theory and gravity," Physics Report, vol. 323, no. 3-4, pp. 183-386, 2000.

[60] P. Burikham, "Magnetic phase diagram of dense holographic multiquarks in the quark-gluon plasma," Journal of High Energy Physics, vol. 2011, no. 5, article 121, 2011.

[61] E. G. Thompson and D. T. Son, "Magnetized baryonic matter in holographic QCD," Physical Review D, vol. 78, no. 6, Article ID 066007, 6 pages, 2008.

[62] F. Preis, A. Rebhan, and A. Schmitt, "Inverse magnetic catalysis in dense holographic matter," Journal of High Energy Physics, vol. 2011, no. 3, article 033, 2011.

[63] P. Burikham and T. Chullaphan, "Magnetized domain walls in the deconfined sakai-sugimoto model at finite baryon density," Journal of High Energy Physics, vol. 2011, article 026, 2011.

[64] G. Lifschytz and M. Lippert, "Holographic magnetic phase transition," Physical Review D, vol. 80, no. 6, Article ID 066007, 7 pages, 2009. 

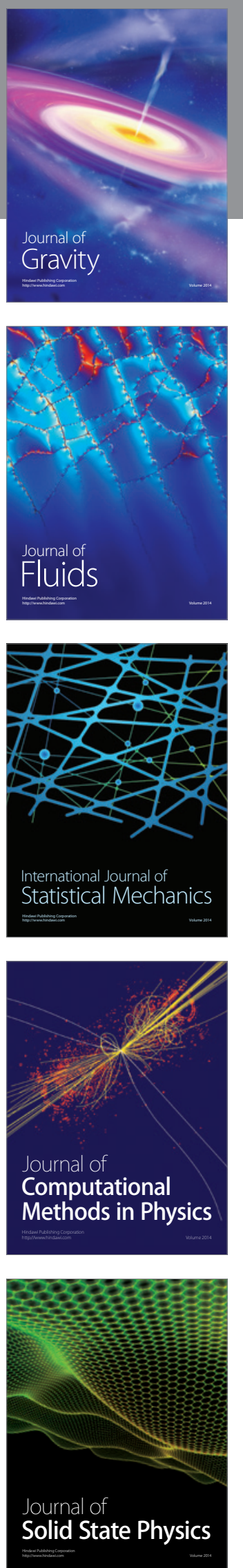

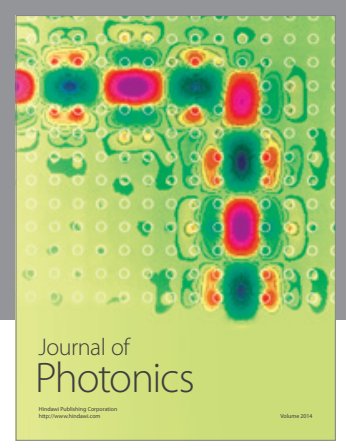

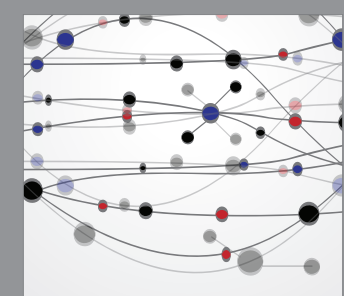

The Scientific World Journal
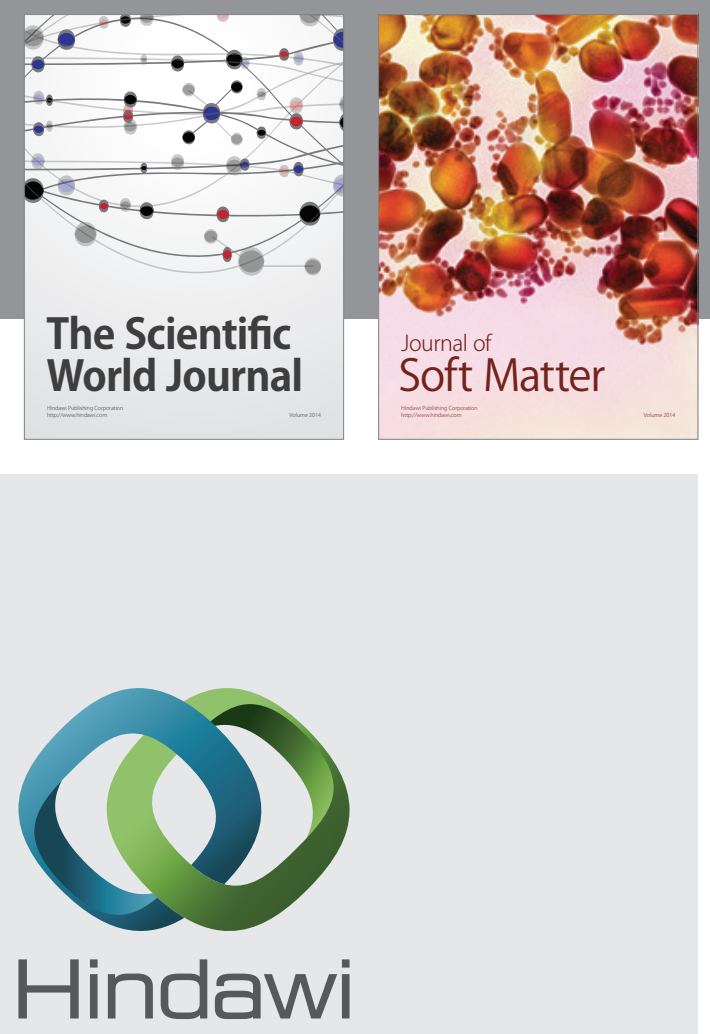

Submit your manuscripts at

http://www.hindawi.com
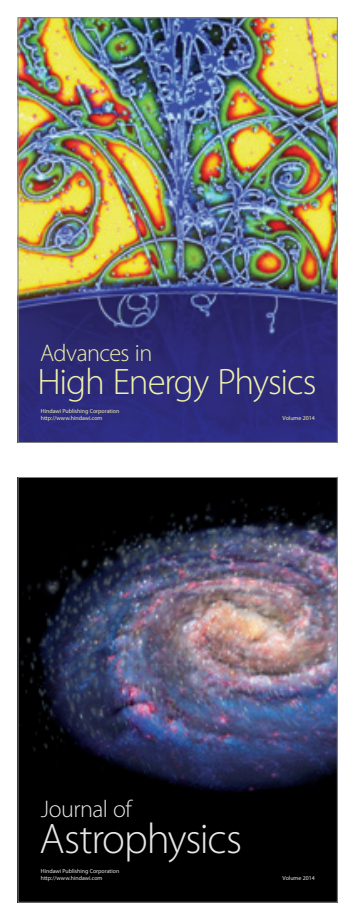
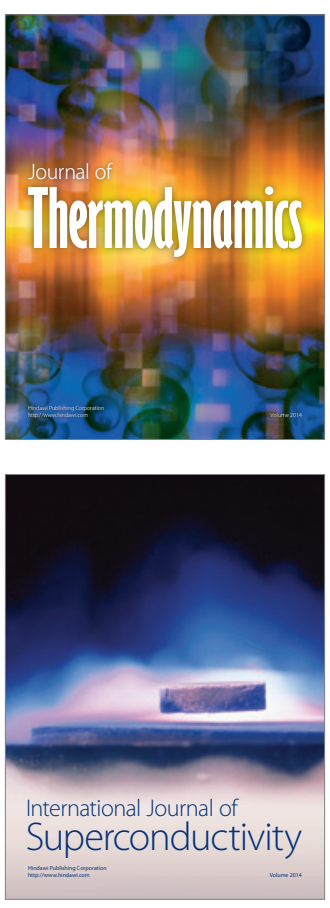
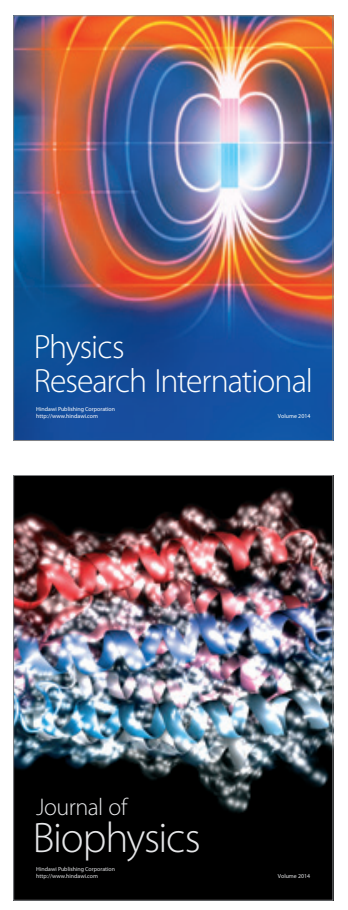
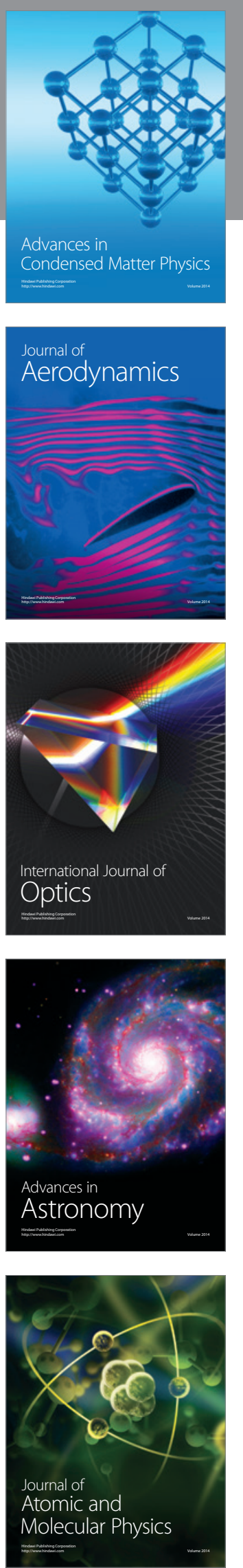